USDA United States

Department of

Agriculture

Index to Selected Science

Forest Service

Pacific Northwest

Publications of the Interior

Research Station

Columbia Basin Ecosystem

Department of the

Interior

Bureau of Land

Management Project

Management

Mar 2000

(iis) 3

tors arith

रुज?

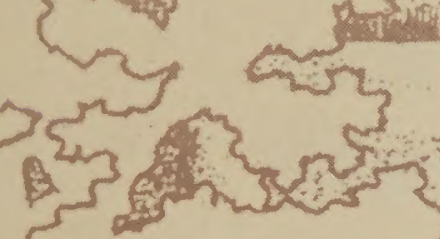

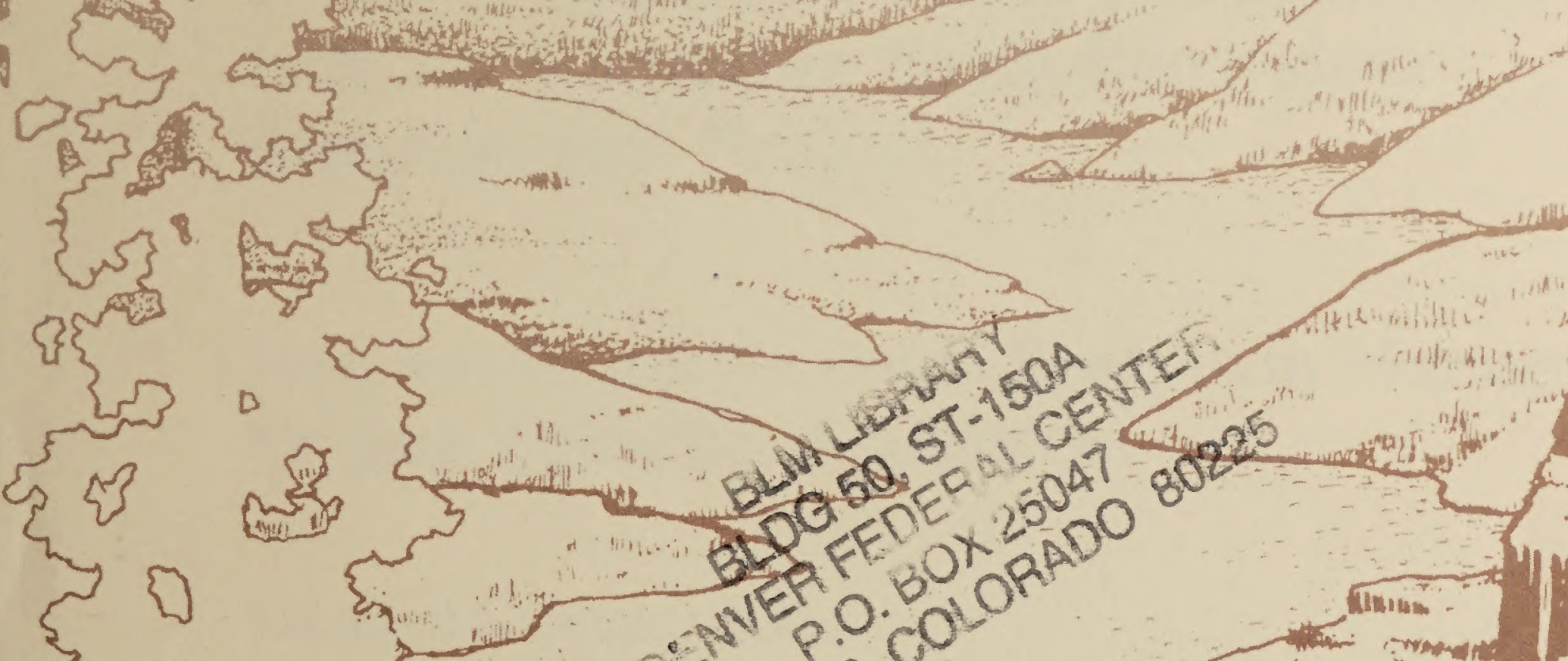




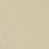




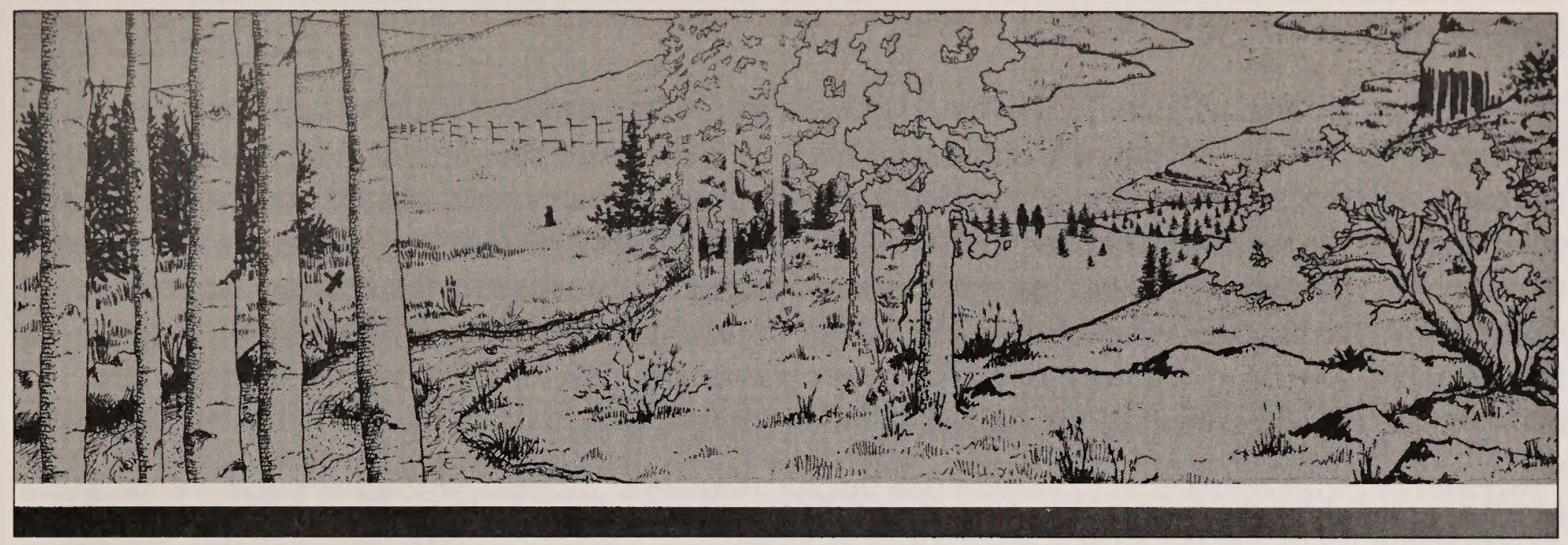

\section{Index to Selected Science Publications of the Interior Columbia Basin Ecosystem Management Project}

\section{Interior Columbia Basin Ecosystem Management Project: Scientific Assessment}

Thomas M. Quigley, Editor

\section{U.S. Department of Agriculture}

Forest Service

Pacific Northwest Research Station

Portland, Oregon

May 2000

BLM Library

Denver Federal Center

Bldg. 50, OC-521

P.O. Box 25047

Denver, CO 80225 


\section{Preface}

The Interior Columbia Basin Ecosystem Management Project was initiated by the USDA Forest Service and the USDI Bureau of Land Management to respond to several critical issues including, but not limited to, forest and rangeland health, anadromous fish concerns, terrestrial species viability concerns, and the recent decline in traditional commodity flows. The charter given to the project was to develop a scientifically sound, ecosystem-based strategy for managing the lands of the interior Columbia River basin administered by the USDA Forest Service and the USDI Bureau of Land Management. The Science Integration Team was organized to develop a framework for ecosystem management, an assessment of the socioeconomic biophysical systems in the basin, and an evaluation of alternative management strategies. This paper is one in a series of papers developed as background material for the framework, assessment, or evaluation of alternatives. It provides more detail than was possible to disclose directly in the primary documents.

The Science Integration Team, although organized functionally, worked hard at integrating the approaches, analyses, and conclusions. It is the collective effort of team members that provides depth and understanding to the work of the project. The Science Integration Team leadership included deputy team leaders Russel Graham and Sylvia Arbelbide; landscape ecology-Wendel Hann, Paul Hessburg, and Mark Jensen; aquatic-Jim Sedell, Kris Lee, Danny Lee, Jack Williams, Lynn Decker; economics - Richard Haynes, Amy Horne, and Nick Reyna; social science-Jim Burchfield, Steve McCool, and Jon Bumstead; terrestrial-Bruce Marcot, Kurt Nelson, John Lehmkuhl, Richard Holthausen, and Randy Hickenbottom; spatial analysis-Becky Gravenmier, John Steffenson, and Andy Wilson.

Thomas M. Quigley

Editor
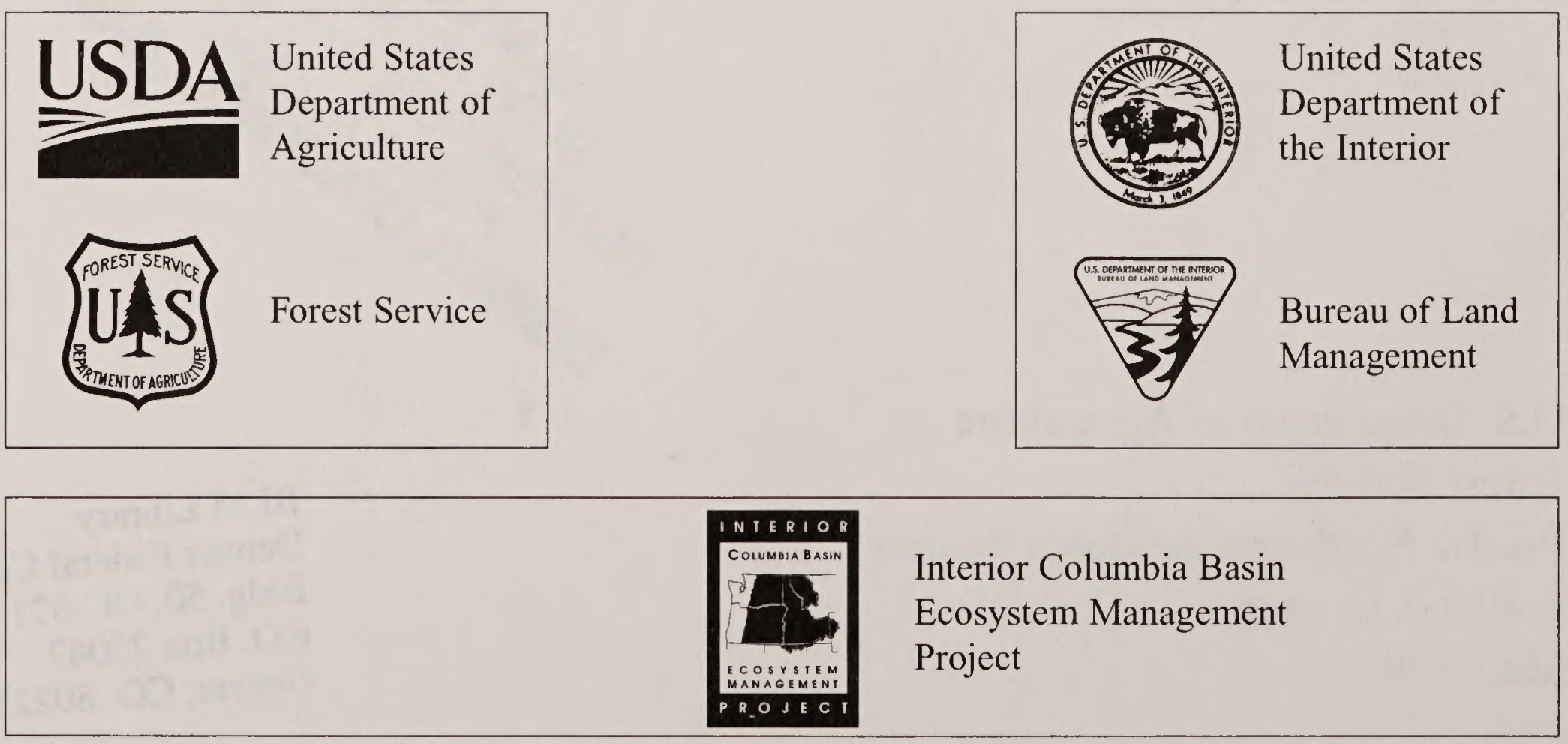


\section{Introduction}

The Interior Columbia Basin Ecosystem Management Project (ICBEMP) is a joint effort of the U.S. Department of Agriculture, Forest Service (FS) and the U.S. Department of the Interior, Bureau of Land Management (BLM). The project is charged with developing a scientifically based ecosystem management strategy for lands administered by the FS and BLM within the interior Columbia River basin (hereafter referred to as the $\operatorname{basin}^{l}$ ). The total area of the basin includes more than 145 million acres ( 58 million ha) of which 76 million acres ( 30 million ha) are administered by the FS and BLM.

The Science Integration Team, the scientific branch of ICBEMP, developed a scientific framework for ecosystem management, conducted a detailed functional assessment, and generated an integrated assessment linking landscape, aquatic, terrestrial, social, and economic characterizations to describe biological, physical, and social systems. This scientific assessment provides research on broad-scale issues in the basin, integrates data across disciplines, and provides both spatial understanding and temporal depth for many critical issues concerning basin ecosystems. The spatial and temporal components of the work enrich our understanding of current and potential future ecosystem conditions as well as the risk and opportunities now present. Land managers are using the science information to develop management strategies and to provide a context for FS and BLM management plans.

\section{Purpose}

This publication provides an easy-to-use, single index to the following major publications of the Science Integration Team:

- A Framework for Ecosystem Management in the Interior Columbia Basin and Portions of the Klamath and Great Basins GTR - PNW-374 (2c.)

- An Assessment of Ecosystem Components in the Interior Columbia Basin and Portions of the Klamath and Great Basins (4 volumes) GTR. PNW - 405

- Highlighted Scientific Findings of the Interior Columbia Basin Ecosystem Management Project

- Integrated Scientific Assessment for Ecosystem Management in the Interior Columbia Basin and portions of the Klamath and Great Basins GTR PNW- 382 (2c.)

- Status of the Interior Columbia Basin: Summary of Scientific Findings GTR

These publications together exceed 2,000 pages in length. Our continuing goal for the scientific assessment is to make the information understandable, accessible, and useful. With this index, we link detailed technical writing with more easily understood highlights and summaries. The abstracts

1 The basin is defined as those portions of the Columbia River basin inside the United States and east of the crest of the Cascade Range, and those portions of the Klamath River basin and the Great Basin in Oregon. 
from these documents are included below for easy reference. A CD-ROM of these documents is available from the Pacific Northwest Research Station. Additional supporting material also is being published. ${ }^{2}$

\section{Abstracts of Indexed Publications}

Haynes, Richard W.; Graham, Russell T.; Quigley, Thomas M., tech. eds. 1996. A framework for ecosystem management in the interior Columbia basin including portions of the Klamath and Great Basins. Gen. Tech. Rep. PNW-GTR-374. Portland, OR: U.S. Department of Agriculture, Forest Service, Pacific Northwest Research Station. 66 p.

A framework for ecosystem management is proposed. This framework assumes that the purpose of ecosystem management is to maintain the integrity of ecosystems over time and space. It is based on four ecosystem principles: ecosystems are dynamic, can be viewed as hierarchies with temporal and spatial dimensions, have limits, and are relatively unpredictable. This approach recognizes that people are part of ecosystems and that stewardship must be able to resolve tough challenges, including how to meet multiple demands with finite resources. The framework describes a general planning model for ecosystem management that has four iterative steps: monitoring, assessment, decisionmaking, and implementation. Because ecosystems cross jurisdictional lines, the implementation of the framework depends on partnerships among land managers, the scientific community, and stakeholders. It proposes that decisionmaking be based on information provided by the best available science and the most appropriate technologies for land management.

Keywords: Ecosystem assessment, ecosystem principles, ecosystem management, planning models, management goals, risk analysis.

Quigley, Thomas M.; Arbelbide, Sylvia J., tech. eds. 1997. An assessment of ecosystem components in the interior Columbia basin and portions of the Klamath and Great Basins. Gen. Tech. Rep. PNW-GTR-405. Portland, OR: U.S. Department of Agriculture, Forest Service, Pacific

Northwest Research Station; U.S. Department of the Interior, Bureau of Land Management. 4 vol. [Quigley, Thomas M., tech. ed.; The Interior Columbia Basin Ecosystem Management Project: Scientific assessment].

These volumes provide detailed information about current conditions and trends for the biophysical and social systems within the basin. This information can be used by land managers to develop broad land management goals and priorities and provides the context for decisions specific to smaller geographic areas. The assessment area covers about 8 percent of the U.S. land area, 24 percent of the Nation's National Forest System lands, and 10 percent of the Nation's BLM-administered lands and contains about 1.2 percent of the Nation's population. This results in a population density less

\footnotetext{
2 Many of the ICBEMP science publications may be ordered by providing your mailing address and the publication title and series (GTR) number to Publication Distribution, Pacific Northwest Research Station, P.O. Box 3890, Portland, OR 97208-3890; phone (503)808-2138; PNW web site: http://www.fs.fed.us/pnw. Related ICBEMP science publications are available through Publications, Ogden Service Center, Rocky Mountain Research Station, 324 25th Street, Ogden, UT 84401-2394; phone (801)625-5437; or email: pubs/rmrs_ogden@fs.fed.us. Information on availability of this material, as well as general information on the Interior Columbia Basin Ecosystem Management Project, is available on the website, www.icbemp.gov.
} 
than one-sixth of the U.S. average. The area has experienced recent, rapid population growth and generally has a robust, diverse economy. As compared to historical conditions, the terrestrial, aquatic, forest, and rangeland systems have undergone dramatic changes. Forested landscapes currently are more susceptible to fire, insect, and disease than under historical conditions. Rangelands are highly susceptible to noxious weed invasion. The disturbance regimes operating on forest and range land have changed substantially, with lethal fires dominating many areas where nonlethal fires historically were the norm. Terrestrial habitats that have experienced the greatest decline include the native grassland, native shrubland, and old forest structures. There are areas within the assessment area that have higher diversity than others. Aquatic systems are now more fragmented and isolated than historically, and the introduction of non-native fish species has complicated current status of native fishes. Core habitat and population centers remain as building blocks for restoration. Social and economic conditions within the assessment area vary considerably, depending to a great extent on population, diversity of employment opportunities, and changing demographics. Those counties with the higher population densities and greater diversity of employment opportunities are generally more resilient to economic downturns. This assessment provides a rich information base, including over 170 mapped themes with associated models and databases, from which future decisions can benefit.

Keywords: Columbia basin, biophysical systems, social systems, ecosystem.

Quigley, Thomas M.; Bigler Cole, Heidi. 1997. Highlighted scientific findings of the Interior Columbia Basin Ecosystem Management Project. Gen. Tech. Rep. PNW-GTR-404. Portland, OR:

U.S. Department of Agriculture, Forest Service, Pacific Northwest Research Station; U.S.

Department of the Interior, Bureau of Land Management. $34 \mathrm{p}$.

Decisions regarding 72 million acres of FS- and BLM-administered lands will be based on scientific findings brought forth in the Interior Columbia Basin Ecosystem Management Project. Highlights of the scientific findings are presented. Project scientists drew three general conclusions: (1) Conditions and trends differ widely across the landscape; as a result, one-size-fits-all strategies will neither effectively restore nor maintain ecosystems. (2) Ecosystem elements are linked to one another; effective ecosystem management requires an understanding of these links. (3) The scientific assessment highlighted a wide variety of risks important to ecological and socioeconomic systems. It also brought forth numerous opportunities to restore ecological systems and provide goods and services. To realize the opportunities, managers must recognize and manage the risks. Three management options were analyzed: current direction, active restoration, and reserve system establishment. Analysis revealed that active restoration was effective in addressing basinwide risks and opportunities.

Keywords: Ecosystem management, ecosystem assessment, ecological integrity, socioeconomic resiliency, risk management.

Quigley, Thomas M.; Haynes, Richard W.; Graham, Russell T., tech. eds. 1996. Integrated scientific assessment for ecosystem management in the interior Columbia basin and portions of the Klamath and Great Basins. Gen. Tech. Rep. PNW-GTR-382. Portland, OR: U.S. Department of Agriculture, Forest Service, Pacific Northwest Research Station. 303 p. (Quigley, Thomas M., tech. ed.; The Interior Columbia Basin Ecosystem Management Project: Scientific assessment).

This document links landscape, aquatic, terrestrial, social, and economic characterizations to describe biophysical and social systems. Integration was achieved through a framework built around six goals for ecosystem management and three different views of the future. These goals are to maintain evolutionary and ecological processes; manage for multiple ecological domains and evolutionary timeframes; maintain viable populations of native and desired non-native species; encourage social and economic resiliency; manage for places with definable values; and manage to maintain a variety of 
ecosystem goods, services, and conditions that society wants. Ratings of relative ecological integrity and socioeconomic resiliency were used to make broad statements about ecosystem conditions in the basin. Currently in the basin, high integrity and resiliency are found on 16 and 20 percent of the area, respectively; low integrity and resiliency are found on 60 and, 68 percent of the area. Different approaches to management can alter the risks to the assets of people living in the basin and to the ecosystem itself. Continuation of current management leads to increasing risks while management approaches focusing on reserves or restoration result in trends that mostly stabilize or reduce risks. Even where ecological integrity is projected to improve with the application of active management, population increases and the pressures of expanding demands on resources may cause increasing trends in risk.

Keywords: Ecosystem assessment, management and goals, ecological integrity, socioeconomic resiliency, risk management.

U.S. Department of Agriculture, Forest Service. 1996. Status of the interior Columbia basin: summary of scientific findings. Gen. Tech. Rep. PNW-GTR-385. Portland, OR: U.S. Department of Agriculture, Forest Service, Pacific Northwest Research Station; U.S. Department of the Interior, Bureau of Land Management. 144 p.

This paper summarizes the scientific findings from the Interior Columbia Basin Ecosystem Management Project. A framework for ecosystem management is described that assumes the broad purpose is to maintain the integrity of ecosystems over time and space. An integrated scientific assessment links landscape, aquatic, terrestrial, social, and economic characterizations to describe the biophysi$\mathrm{cal}$ and social systems. The status of ecosystems is described in terms of current conditions and trends under three broadly defined management options. The scientific information brought forward will be used in decisionmaking and may amend FS and BLM plans within the basin. The information highlighted here represents an integrated view of biophysical and socioeconomic elements at a scale never before attempted. The risks and opportunities are characterized in the broad context of the basin for managers and the public to use as a foundation for discussion about future management.

Keywords: Ecosystem assessment, ecosystem management, ecosystem integrity, risk analysis. 


\section{Index}

This index is not intended to be a comprehensive listing of all occurrences of the following terms. Rather, it is meant as an aid in locating discussions pertinent to the variety of concepts listed. Please use this index as a way to access the rich discussion available in the publications themselves. The individual volumes are designated as follows, and page numbers refer only to the volumes they follow.

- F A Framework for Ecosystem Management in the Interior Columbia Basin and Portions of the Klamath and Great Basins (PNW-GTR-374)

- I Integrated Scientific Assessment for Ecosystem Management in the Interior Columbia Basin and portions of the Klamath and Great Basins (PNW-GTR-382)

- C (Vol no.) An Assessment of Ecosystem Components in the Interior Columbia Basin and Portions of the Klamath and Great Basins (4 volumes) (PNW-GTR-405)

- S Status of the Interior Columbia Basin: Summary of Scientific Findings (PNW-GTR-385)

- H Highlighted Scientific Findings of the Interior Columbia Basin Ecosystem Management Project (PNW-GTR-404)

\section{A}

Abiotic -

$\mathrm{F}-18$

$\mathrm{C}(\mathrm{Vol} \mathrm{1})-127$

$\mathrm{C}($ Vol 2) - 365, 368, 371, 373, 375, 382, 388-389, 517

C (Vol 3)- 1081, 1543, 1618, 1649

Accuracy/Map accuracy-

C (Vol 4)- 2065

Adaptive management-

$\mathrm{F}-3,5,11,13,22,24-25,27,33,36,45$, Appendix B

I- $15,20,27,142,163,170-171,173,183$, Appendix A

$\mathrm{S}-25-26,92,138$

$\mathrm{C}(\mathrm{Vol} 1)-8,12,23,53,92$

$\mathrm{C}($ Vol 3) - 1374, 1380, 1652, 1654

Adfluvial-

$\mathrm{C}(\mathrm{Vol} 4)-1974,1990,2052$

Aeolian-

C (Vol 3) - 1176, 1182, 1190-1191, 1194, 1198, 1210, 1212, 1237, 1288, 1431-1432

C (Vol 1)- 36-38, 40-41, 124-126, 146, 245-246, 248-249, 254-256, 309

Air mass-

C (Vol 3)- 1533

C (Vol 1)- 185, 188, 193, 309-312

Air quality-

$\mathrm{F}-24$

$\mathrm{S}-94-95$

C (Vol 1)- 76, 198, 200

C (Vol 2)- 401, 804, 873, 875-877, 928

$\mathrm{C}(\mathrm{Vol} 3)-1536,1676$

C (Vol 4)- 1911, 1964, 1984, 2024, 2031 
Alevin-

C (Vol 3)- 1101, 1182, 1431

Alkali/Alkalinity-

C (Vol 1)- 309

C (Vol 3)- 1088, 1100, 1191, Appendix 4A, 1538, 1540-1541, 1618-1620, 1623

Allopatric-

C (Vol 3) - 1149, 1201, 1203 (Map 4.17), 1204-1207, 1209 (Map 4.19), 1210, 1213, 1245-1246, 1261, 1431, Appendix 4D

Allospecies-

C (Vol 3)- 1431, 1648

Alluvium-

C (Vol 1) - 37-42, 115, 120, 124-127, 143, 146-148, 177, 211, 247-251, 254-257, 309

$\mathrm{C}(\mathrm{Vol} 3)-1082,1127,1135$, Appendix 5F

Altered sagebrush steppe (sagebrush grassland) -

$\mathrm{S}-74,78$

$\mathrm{C}($ Vol 1) - 202

C (Vol 2)- 765-767, 776, 778, Appendix 3F

C (Vol 3) - Appendix 4A, 1536, 1603-1607, 1620, 1626

Amenities-

$\mathrm{F}-4,9,46$

I- $35,76,86,124,127,163$

$\mathrm{S}-23,43,46,52,57-58,129$

C (Vol 1)- 20, 25, 53, 83, 87, 89-90

C (Vol 2) - 364, 447-448, 883, 907-908, 910, 917-918, 924-925, 965

C (Vol 4) - 1732, 1735, 1737, 1805, 1807, 1812-1813, 1817, 1832, 1837, 1881, 1897-1899, $1905,1919,1935,1939,1643-1950,1957-1960,1964,1966-1968,1971$

American Indians (see also: Tribal/Tribes) -

$\mathrm{F}-4-5,16,18,22,27$

$\mathrm{H}-25$

I- $11,21,26,29,34,36,38,52-53,56,75,91,117,145,177-178,185$

$\mathrm{S}-22,26,29-30,35,46-47,61-66,75,93-95,97,139,142$

C (Vol 1) - 19-20, 73-74, 76, 78, 84, 87-88, 91

C (Vol 3) - 1118, 1279, 1318, 1320, 1507-1508, 1521, 1531, 1533-1534, 1536, 1543, 1548, $1574,1579,1581,1644$

C (Vol 4) - 1745, 1747, 1799, 1838, 1878-1880, 1883-1884, 1889-1890, 1893-1894, 1907 , 1914-1915, 1921, 1922 (Map 7.2), 1923-1932, 1985, 2028, 2034

Ammocoetes-

Amphibians-

C (Vol 3)- 1270-1271, 1275, 1431

$\mathrm{H}-21$

I- 58, 76, Appendix C, Appendix D, Appendix E

S- 94, 97, 99

C (Vol 1)- 74-75, 77, 333

C (Vol 2)- 776, 875, Appendix 3J, Appendix 3M

C (Vol 3) - 1107, 1249, 1356, 1365, Appendix 4A, 1507, 1509-1511, 1518, 1522-1523, $1531,1534,1538,1540,1556,1568-1569,1576,1582,1584-1586,1591,1600$, $1608,1610,1612,1618,1621-1622,1637-1638,1640,1645-1646,1654-1656$, Appendix $5 \mathrm{H}$

C ( Vol 4) - 2028, 2033 
Anadromous fish (see: Fish, anadromous)

Antelope (see: Pronghorn antelope)

Anticyclone-

C (Vol 1) - 188, 309, 313

Aquatic ecology/Aquatic findings-

I- 146-147, 183

$\mathrm{S}-139$

C ( Vol 2)- 920

C (Vol 3) - Chapter 4 Aquatic Species and Habitats

Aquatic ecosystem integrity/Aquatic ecosystem health/Aquatic ecosystem function -

I- $146,148,170$

$\mathrm{S}-101,139$

C (Vol 1)- 65-66

C (Vol 3)- 1087, 1107, 1113, 1142, 1360, 1339, 1357, 1363

Aquatic habitat-

I- 164,171

S- 104-105, 107

C (Vol 1)- 69, 114, 184

C (Vol 3) - 1110, 1253, 1335, 1347, 1374, 1377, 1582

$\mathrm{C}(\mathrm{Vol} 4)-1799$

Aquatic integrity -

$\mathrm{H}-10$

I- 103, 104 (Figure 37), 108-110, 113, 116, 120, 123, 134, 146, 170, 172

S- $105,111,116,119,121-123,125-128,139$

C (Vol 1)— $\quad 66,69-70,116,118$

$\mathrm{C}(\mathrm{Vol} 2)-\quad 961$

C (Vol 3) - 1253, 1289, 1339, 1347, Appendix 4C

C (Vol 4)_ 2043

Aquatic priority areas -

$\mathrm{I}-120$

C (Vol 1) - $\quad 10$

C (Vol 3) - $\quad 1141,1176,1237,1291,1348-1352,1356,1361,1363-1364,1374-1375$

C (Vol 4)_ $\quad 1890,1914,1926,2026,2032$

Aquatic species (see also: Fish; Salmonids, key) -

$\mathrm{H}-11$

$\mathrm{I}-24,103$

$\mathrm{S}-68,93-94,101$

C (Vol 1)- 16, 20, 51-53, 63, 67, 74, 175, 183

$\mathrm{C}$ (Vol 3)- Aquatics-Chapter 4

C (Vol 4)- 1929, 2031

Aquatic strategy-

$\mathrm{F}$ - Appendix B

$\mathrm{H}-34$

I- $15-16,117,142,146-148,153,163,170,172,184$, Appendix A

S- 113,140

C (Vol 1) - 10, 12, 16, Appendix 1A, 63, 78

C (Vol 3) - 1074, 1083, 1137, 1139, 1182, 1190, 1211, 1220, 1233, 1363-1264, 1267 , $1340-1341,1347-1348,1354,1356,1365,1370-1373,1375$

C (Vol 4)- 1801,1829 
Arthropod-

$\mathrm{S}-97$

$\mathrm{C}(\mathrm{Vol} \mathrm{1})-77,78$

C (Vol 3) - 1556-1560, 1563, 1565-1566, 1612, 1652, 1654

Attitudes, beliefs, and values (see also: Culture/Cultural; Economic'value)-

$\mathrm{F}-20$

S- 34, 37-38

C (Vol 1)- 20,61, 87-89, 91-92, 105

C (Vol 3)- 1345

C (Vol 4) - Chapter 7 Social Assessment, 1887-1881, 1888-1890, 1892, 1898,1901-1920, 1925, 1927, 1928, 1930-1934, 1936-1940, 1943, 1946, 1948-1953, 1956, 1958, $1960,1961,1962,1990$

Attribute (geographic) -

C (Vol 4)- 2022, 2029, 2031, 2033-2037, 2041, 2045, 2049, 2055, 2057, 2065-2066

B

Bears, general-

black-

C (Vol 4)- 1929

I- 51, 131, Appendix E

$\mathrm{C}(\mathrm{Vol} 1)-331$

C (Vol 3)- 1572-1573, 1575, 1579-1580, 1584, 1614, 1646

grizzly-

$\mathrm{F}-22$

I- 37, 51, 108, 131, 145, 171, Appendix D, Appendix E

$\mathrm{C}(\mathrm{Vol} 1)-12,332$

$\mathrm{C}(\mathrm{Vol} 2)-390$

C (Vol 3) - 1513, 1533, 1572-1573, 1575, 1577-1581, 1583-1584, 1614

$\mathrm{C}(\mathrm{Vol} 4)-1724,1787,1817$

Biodiversity (Biological diversity)-

F- 10, 18, 22, 45, Appendix B

I- 14, 34, 56, 74-75, 78 (Figure 22), 79 (Figure 23), 140, 166-167, 170, 181, 183, Appendix A

S- 93, 98 (Map 14), 99-100, 134

C (Vol 1) - 12, 23, 73, 77-78, 79 (Map 1.9)

C (Vol 2) - 363-365, 383, 385, 388, 390, 392, 579, 611, 621, 664, 768, 775-776, 785, 795, $805,893,897,918,925,961,965$

C (Vol 3)- 1074, 1101, 1263, 1348, 1354, 1365, 1370-1371, 1375, 1378, 1507-1508, 1512, $1516,1527-1533,1543,1554,1556,1563,1615,1618,1621-1623,1624$ (Map 5.4), 1626, 1643-1644, 1646, 1648

C (Vol 4)- 1905, 2028, 2044

Biogeochemical/Biogeochemical processes-

C (Vol 1)- 56,61

$\mathrm{C}(\mathrm{Vol} 2)-365,375,382-383,385,388-389,404-405,420,465-468,470,512-517,554$, $572,602,644,667-668,670-671,845,867,873,879,883,924-925,961$

Biogeograph-

C (Vol 2)- 367, 383, 961

C (Vol 3)- 1249, 1561, 1627-1628 


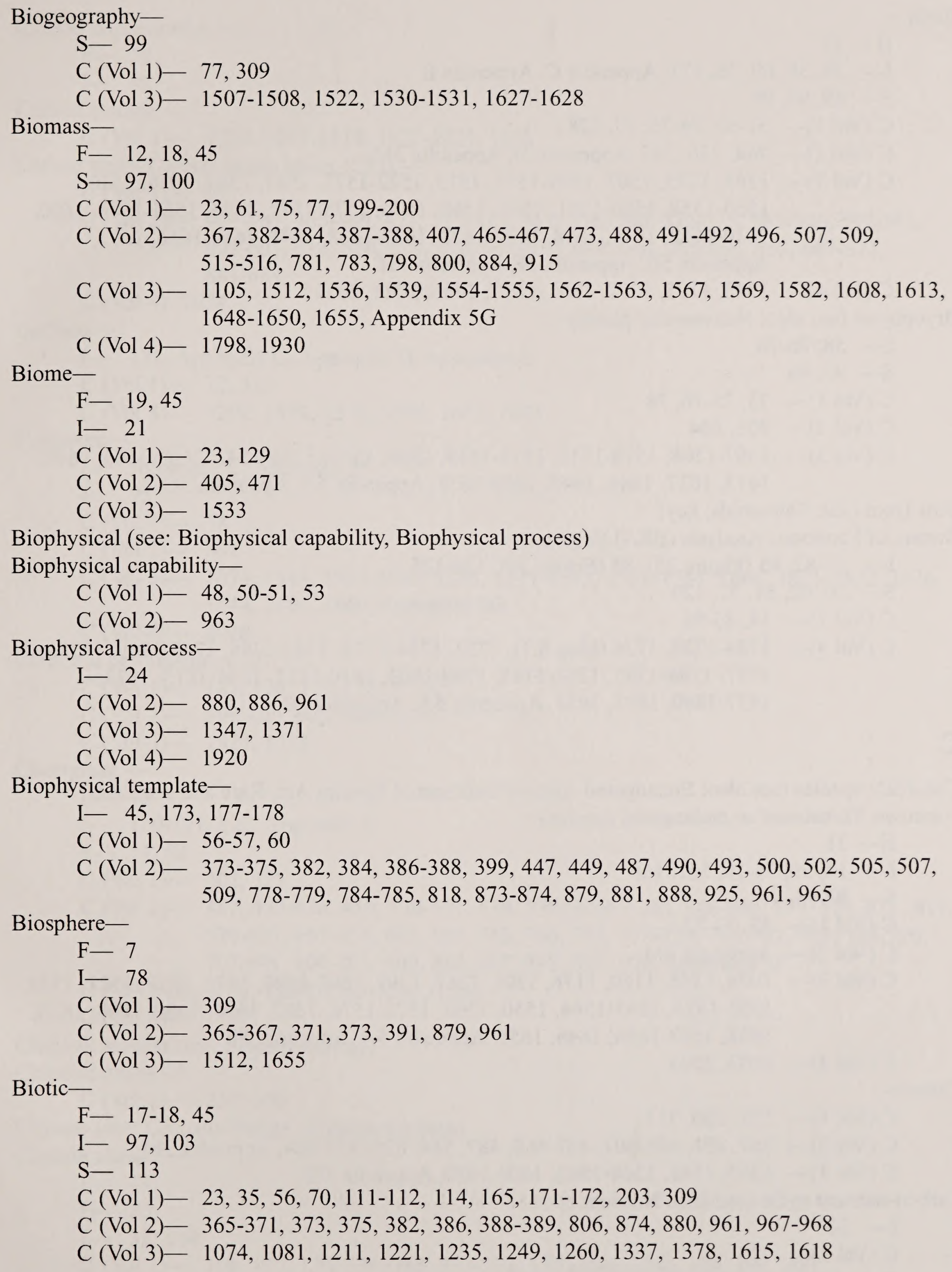


Birds-
$\mathrm{H}-21$
I- $34,58,60,76,171$, Appendix C, Appendix E
$\mathrm{S}-89,94,99$
C (Vol 1) - 51-52, 74-75, 77, 328
C (Vol 2) - 764, 776, 797, Appendix 3J, Appendix 3M
C (Vol 3) - 1365, 1375, 1507, 1509-1511, 1513, 1522-1523, 1531, 1534, 1536, 1540, $1556-1558,1560-1561,1563,1568,1570,1575-1577,1583,1585-1586,1600$, 1610-1612, 1614, 1618, 1620-1622, 1637-1638, 1645, 1654-1656, 1675, Appendix 5E, Appendix 5F, Appendix 5H
C (Vol 4)— 1743, 1775, 1777, 1801, 1817, 1986, 2028, 2033

Bryophytes (see also: Nonvascular plants)_-

I- $58,75-76$

S- $-93,96$

C ( Vol 1) - 73, 75-76, 78

$\mathrm{C}(\mathrm{Vol} 2)-801,804$

C (Vol 3) - 1507-1508, 1510-1511, 1518-1519, 1529, 1531, 1536-1542, 1600-1606, 1610, $1613,1637,1644,1648,1650-1651$, Appendix 5A, Appendix 5G

Bull Trout (see: Salmonids, key)

Bureau of Economic Analysis (BEA) Region-

I- 81-82, 83 (Figure 25), 85 (Figure 26), 124-125

$\mathrm{S}-20,42,51,57,129$

C (Vol 1)- 14, 83-86

C (Vol 4) - 1724-1725, 1726 (Map 6.1), 1732, 1734-1735, 1743-1744, 1767-1769, 1775, $1777,1784-1785,1793-1795,1799-1803,1810-1812,1814-1815,1833$, 1837-1840, 1897, 1935, Appendix 6A, Appendix 6B

\section{C}

Candidate species (see also: Endangered species/Endangered Species Act; Rare and sensitive species; Threatened or endangered species) -

$\mathrm{H}-21$

I- $14,59,75-76,161,166$

$\mathrm{S}-93-95,97$

C (Vol 1)- 47, 73-77

C (Vol 2) - Appendix 3M

C (Vol 3) - 1074, 1145, 1160, 1176, 1201, 1267, 1303, 1507-1508, 1511, 1519, 1521, 1523, $1533-1534,1543-1544,1550,1568,1575-1576,1587,1591,1600,1608,1628$, 1638, 1643-1644, 1646, 1655, Appendix 5A, Appendix 5E

Carbon-

C (Vol 4) - 2033, 2044

C (Vol 1) - 127, 200, 311

C (Vol 2)- 367, 391, 405-407, 467-468, 487, 514, 876, 923-924, Appendix 3M

C (Vol 3) - 1365, 1561, 1564-1565, 1608-1610, Appendix 5G

Carbon-nutrient cycle (see also: Nutrient cycle) -

$\mathrm{I}-30$

$\mathrm{C}(\mathrm{Vol} 1)-57$

C (Vol 2) - 365-369, 371-373, 386, 389-391, 404-407, 446-448, 467-468, 487, 879, 910, 917-918, 920, 923-925, 963, 965, Appendix 3M

C (Vol 3) - 1562, 1608-1610, Appendix 5G 
Carbon sequestration -

$$
\begin{aligned}
& \mathrm{F}-22 \\
& \mathrm{I}-38
\end{aligned}
$$

Carbon storage -

C (Vol 4)- 1724, 1817-1818, 1822-1823, 1828

Carbon stress/Carbon Stress Index (CSI) -

C (Vol 1)- 61

C (Vol 2) - 405-407, 465-469, 471, 512-517, 531, 556, 560, 572-573, 585-586, 592-593, $602-603,610,620,624,626,635-636,644,646,655-656,663,667-672$, 883-884

Caribou-

C (Vol 4)- 2024

I- 171, Appendix C, Appendix D, Appendix E

C (Vol 1)- 12, 333

Carnivore-

$$
\text { C (Vol 3) - 1292, 1574, 1578, 1600, 1641, } 1645
$$

I- Appendix C, Appendix D

$\mathrm{S}-95$

C (Vol 1)- 78

C (Vol 2)- 391

C (Vol 3) - 1074, 1513, 1522-1523, 1531, 1571-1575, 1579-1581, 1600, 1621-1622, 1626, 1644, 1656, 1660, Appendix 5G

C (Vol 4)- 2028

Cell/Grid cell (geographic) -

C (Vol 1) - 123, 128, 171-172

C (Vol 2)- 399-400, 405, 436

C (Vol 3)- 1523, 1628

Cheatgrass -

$\mathrm{H}-15$

I- 120-121, 123, Appendix E

$\mathrm{S}-51,82$

C (Vol 1)- 202, 321

C (Vol 2) - 387, 433-436, 459, 530-531, 536, 539 (Map 3.26), 548-549, 572, 579, 611, 615, $620-621,655-656,663,697,763,766,776,778-779,781-782,785,788-790$, $797-801,804-805,809,882,887,893,897,928$, Appendix 3I

$\mathrm{C}($ Vol 3) - 1537, 1559, 1565-1567, 1586

$\mathrm{C}(\mathrm{Vol} 4)-1887$

Chinook salmon (see: Salmonids, key)

Chinook winds -

C (Vol 1)- 234, 309

Climate (see: Climate change; Climate division)

Climate change -

$\mathrm{F}-19$

$\mathrm{H}-20$

I- 32, 164

C (Vol 1) - 108, 127, 130, 136, 165, 171-172, 174 (Map 2.24), 189, 198, 200

$\mathrm{C}(\mathrm{Vol} 2)-369,386,784,873,876,886$, Appendix 3M

C (Vol 3)- 1176, 1182, 1184, 1199, 1212, 1261, 1263 
Climate division -

C (Vol 1)- 235, 309

C (Vol 2)- 440, 498, 504, 530, 556, 610, 655, 663, 808

Clusters (see: Forest clusters; Range clusters; Social/economic clusters)

Coarse woody debris -

$\mathrm{S}-74$

C (Vol 1) - 201-202, 205-206

C (Vol 3) - 1234, Appendix 5F

Cold forest potential vegetation group (PVG) (see: Potential vegetation group, cold forest)

Collaborative/Collaboration -

$\mathrm{H}-3$

I- 170

$\mathrm{S}-26,59$

C (Vol 1)- 53, 91-92, 116, 172

$\mathrm{C}(\mathrm{Vol} 3)-1378$

C (Vol 4) - 1908, 1918-1919, 1971-1972, 1974-1976, 1982, 1984, 1988-1989

Colluvium -

C (Vol 1)- 146, 250, 252, 309

$\mathrm{C}(\mathrm{Vol} 3)-1135,1366$

Columbia River basin succession model (CRBSUM) -

C (Vol 1) - 127, 152

C (Vol 2)- 339, 408, 411, 432, 436, 441-442, 447, 846, 849, 852, 961-962, Appendix 3M

C (Vol 4) - 1724, 1786, 1832, 2018, 2021, 2024-2025, 2029, 2031, 2033, 2035, 2037, 2040-2043, 2045, 2051, 2054, 2058

Community (see: Community resiliency; Community stability)

Community resiliency (see also: Economic resiliency; Resiliency; Social resiliency)-

$\mathrm{F}-21$

I- 125,161

$\mathrm{S}-43,45,141$

C (Vol 1)- 13, 89-90

C (Vol 4)— 1894, 1938-1939, 1942-1950, 1953, 1955-1956

Community stability -

$\mathrm{F}-21$

$\mathrm{S}-56-57$

C (Vol 1)- 84

C (Vol 4) - 1724, 1728, 1767, 1805-1806, 1808, 1838, 1936-1938, 1955, 1975

Composite ecological integrity -

$\mathrm{H}-8$

I- 109-110, 122 (Figure 40), 123-124, 135, 137, 149, 153, Appendix B

S- 118 (Map 18), 119, 121, 125, 133 
Connected/Connecting/Connectivity -

$$
\begin{aligned}
& \mathrm{H}-11 \\
& \text { I- 16, 60-61, 97, 103, 108, 113-117, 119, 140, 149, 161, 166-167, 169-171, 174, } 184 \\
& \text { S- } 68,115,127-128,134,141 \\
& \text { C (Vol 1)- 48, 51-53, 65, 70-71, 183, } 250 \\
& \text { C (Vol 2) - 373, 392-393, 431-432, 512, 746, 877, 885, 901, 903, 905, 916, 918, 961, 963, } \\
& \text { 967-968 } \\
& \text { C (Vol 3) - 1084-1085, 1110, 1112-1113, 1142, 1149, 1173, 1184, 1212, 1223, 1232, 1234, } \\
& 1236,1241,1260-1261,1278,1339-1340,1354,1356-1357,1359,1361,1363 \text {, } \\
& \text { 1365, 1376, Appendix 4D, Appendix 4G, 1573, 1580, 1627, } 1646 \\
& \text { C (Vol 4)- 1808, 1944-1945, 1977, 1986, } 2066
\end{aligned}
$$

Continental air mass -

\section{Convection -}

$$
\text { C (Vol 1)- 193, } 309
$$

$$
\text { C (Vol 1)- } 310
$$

Convective precipitation -

$$
\text { C (Vol 1)- 185, 194, 231, 235, } 310
$$

Cool shrub potential vegetation group (PVG) (see: Potential vegetation group, cool shrub)

Cooperative observer network (COOP) -

$$
\mathrm{C}(\text { Vol 1) - 188, 235, 237, } 310
$$

Cultural resources -

$$
\begin{aligned}
& \mathrm{H}-28 \\
& \mathrm{I}-138 \\
& \mathrm{~S}-134
\end{aligned}
$$

Culture/Cultural (see also: Attitudes, beliefs, and values; Economic value)-

F- 8, 9, 15-17, 20-21, 23, 26, Appendix B

I- $12,16,19,21,22,29,35-36,38,41,52,74,199,124,138,148,163,167,171$, 177-178, 184, 187, Appendix A

S- $18,29,47,61-63,129,134$

C (Vol 1)- 84, 87, 91, 98

C (Vol 2)- 384, 433, 488, 512, 923

C (Vol 3) - 1073, 1106-1107 (cultural fish stocks), 1146, Appendix 4A, 1511, 1519, 1521, $1543,1550,1652$

C (Vol 4) - 1743, 1746, 1749, 1812, 1832, 1839, 1877, 1879, 1883-1884, 1903, 1907-1908, 1919-1921, 1923, 1925-1932, 1934, 1943, 1951, 1953, 1955, 1960-1961, 1968, 1971,1983

Cumulative effects -

F- 19, Appendix B

$\mathrm{H}-12$

I- 115, Appendix A, Appendix C

$\mathrm{S}-75,82,104,137$

C (Vol 1)- 70

C (Vol 2) - 390, 393, 462

C (Vol 3) - 1135, 1146, 1234, 1265, 1342, 1354, 1370-1371

Cyclone-

$$
\mathrm{C}(\mathrm{Vol} 4)-1814
$$

C (Vol 1) - 310 
D

Dams-

$\mathrm{H}-17$

I- $35,51-52,73,100,103,117,123,166,171,173$

S- $13,31,37,46-47,75,99,104,107,113,116$

C (Vol 1)- 18, 43, 57, 63, 67, 71, 77, 88, 145, 164, 259-260, 269, 274, 276, Appendix 2A

$\mathrm{C}(\mathrm{Vol} 2)-479,507,509,768,823$

C (Vol 3) - 1074, 1085, 1093, 1094 (Map 4.3), 1095-1096, 1112, 1115, 1124-1125, 1162, $1173,1184,1199,1213,1221-1223,1228,1234-1236,1254,1264,1268$, $1270-1271,1279,1285,1292,1314,1318,1337,1342,1344-1345,1357$, 1375-1376, Appendix 4G, 1582, 1646

C (Vol 4)- 1746-1749, 1766, 1784, 1801, 1885, 1889-1890, 1914, 1920, 2025, 2031-2032

Databases-

F- 29

$\mathrm{H}-34$

I- $15,22,75,175,179-180,183$, Appendix E

S- 93

C (Vol 1)- 13, 20, 71, 73, 75, 96-98, 116, 123

$\mathrm{C}($ Vol 2) - 433

C (Vol 3)- 1093, 1094 (Map 4.3), 1095, 1102-1103, 1118, 1146-1149, 1174, 1378, Appendix 4C, Appendix 4D, 1511, 1517, 1521-1522, 1527, 1529, 1531, 1568, $1643,1646-1647,1651-1652,1676$

C (Vol 4)- 1880, 1984, 2018, 2020, 2032, 2037, 2039, 2043-2045, 2055, 2057-2058, 2060

Data layer/Layer-

$\mathrm{C}(\mathrm{Vol} 1)-96$

C (Vol 4)— 2018, 2031, 2039, 2051, 2054, 2065

Data set/Geographic data set-

C (Vol 1)- 97, 137, 196, 214

C (Vol 2) - 395, 404, 435, 756, Appendix D

$\mathrm{C}(\mathrm{Vol} 3)-1163,1167-1168,1368$

C (Vol 4)- 1810, 2031, 2033-2036, 2048-2050, 2054-2057, 2065

Decisionmaker/Decisionmaking-

F- 3-5, 7-11, 13, 15, 20, 23, 26-29, 33, 45 (definition), Appendix B

I- $19,27,43,138,145$, Appendix A

S- $24,58,65,66,134,139$

$\mathrm{C}(\mathrm{Vol} 1)-18,23,172$

$\mathrm{C}($ Vol 2) -779

C (Vol 4) - 1816, 1899, 1906, 1919, 1927, 1931-1932, 1936, 1955, 1971-1972, 1975, 1979, $1980,1982,1988,1989$

Decreaser species-

I- Appendix D

C (Vol 2)- 962

$\mathrm{C}(\mathrm{Vol} 3)-1589$ 
Deer-

$\mathrm{F}-22$

I- 51, 130-131, Appendix E

S- 49

C (Vol 1)- 332-333

C (Vol 2)- 776, Appendix 3J

C (Vol 3) - 1536, 1563, 1574, 1577, 1581, 1601, 1610, 1640

C ( Vol 4)- 1887, 1929

Demographic-

F- 20

I- $33,127,166$

$\mathrm{S}-65$

C (Vol 3)- 1113, 1221, 1261, 1676

C (Vol 4) - 1729-1732, 1737, 1743, 1749, 1893-1900, 1931, 1936, 2025, 2031-2032, 2045

Derived data-

$\mathrm{C}(\mathrm{Vol} \mathrm{1})-96$

C (Vol 4)- 2037, 2050, 2065

Desired future conditions-

$\mathrm{F}-45$

$\mathrm{C}($ Vol 1$)-23$

Detritivory-

S- 97

$\mathrm{C}(\mathrm{Vol} 1)-76$

C (Vol 3) - 1554-1556, 1563, 1564-1565, 1675 (definition)

Dewater/Dewatering-

$\mathrm{C}(\mathrm{Vol} 2)-962$

$\mathrm{C}(\mathrm{Vol})-1376$

Digitize-

$\mathrm{C}(\mathrm{Vol} 4)-2065$

Disseminule

$\mathrm{C}(\mathrm{Vol} 3)-1675$

Disturbance-

F- $10,11,17-18,23,32-34$

I- $31,45,60-62,68,100,103,108,138,140,142-143,149,165-166,169-170,172$, $178-180,184-185$

S- $34,37,49,51,67,75-91,92,134-136,142$

C (Vol 1) - 14, 16, 23 (definition), 35-36, 43, 50-71, 190-195, 205, 259-276

C (Vol 2) - Chapter 3 Landscape Ecology of the Basin, 376-377, 401-403, 420, 437-441, $468-470,471-478,667-672,805-806,883,905$

C (Vol 3) - 1081-1082, 1101, 1110-1112, 1173, 1183, 1184, 1221, 1239, 1253, 1261-1265, $1341-1342,1356-1360,1370-1374,1530,1535,1555,1584,1601,1619-1620$

C (Vol 4)- 1910, 2031-2032, 2037, 2039-2045, 2051

Disturbance regime (succession/disturbance regime) -

C (Vol 2) - 386-399, 421, 424, 427, 441, 446-447, 460-465, 473, 475, 477, 496, 508, $511-516,795,816-828,906,909-915,962$ (definition) 
Domains-

$$
\begin{aligned}
& \text { F- 18-19 } \\
& \text { I- } 12,30,32-33,96 \\
& \text { S- } 24 \\
& \text { C (Vol 1)- } 118
\end{aligned}
$$

Down burst-

$$
\text { C (Vol 1)- 234, } 310
$$

Down wood-

$$
\mathrm{S}-94,97
$$

$\mathrm{C}($ Vol 1$)-75,77$

C (Vol 2)- 492

C (Vol 3) - 1557-1558, 1563, 1568-1569, 1600, 1608, 1614, 1647, 1656, 1658, 1676, Appendix 5F, Appendix 5G

Dry forest potential vegetation group (PVG) (see: Potential vegetation group, dry forest)

Dry grass potential vegetation group (PVG) (see: Potential vegetation group, dry grass)

Dry shrub potential vegetation group (PVG) (see: Potential vegetation group, dry shrub)

\section{E}

Eagle-

bald-

I- 51, Appendix C, Appendix D, Appendix E

C ( Vol 1)- 328

C (Vol 3)- 1533, 1576, 1578, 1608

$\mathrm{C}(\mathrm{Vol} 4)-1787$

golden-

C (Vol 1)- 329

C (Vol 2)- Appendix 3J

$\mathrm{C}(\mathrm{Vol} 3)$ - Appendix 5E

Earthquake-

C (Vol 1)- 116, 181

C (Vol 3)- 1082

$\mathrm{C}(\mathrm{Vol} 4)-2027$

Ecological disequilibria/Ecological disequilibrium (see also: Equilibria/Equilibrium) $\mathrm{C}(\mathrm{Vol} 2)-372-373,924,963$

Ecological function (see: Key ecological function)

Ecological integrity/Ecological ratings-

$\mathrm{F}-17,33,35$

$\mathrm{H}-6,8-9,12-13,28,30,32,34$

I- 12-16, 30-32, 34, 39-40, 95-97, 105, 113-114, 123, 130-132, 134-135, 137, 140, 149 , 150 (Figure 49), 151 (Figure 50), 152 (Figure 51), 153-157, 161, 167, 181, 184-185, 187, Appendix B

S- 115, 119, 126-128, 133-134, 140-142

C (Vol 1)- 11, 16, 18, 62, 78

C (Vol 2) - 365, 381 (Map 3.6), 390, 413, 416 (Map 3.8), 417 (Map 3.9), 532 (Map 3.22), 533 (Map 3.23), 838, 841, 900, 921, 923-925, 961, 963, Appendix 3M

C (Vol 3) - 1085, 1342

C (Vol 4)- 1810, 2026 
Ecological predictability-

C ( Vol 2)- 963

Ecological process/Ecological process interpretations-

I- 30-32, 180

C (Vol 1) - 107, 149, 151

$\mathrm{C}(\mathrm{Vol} 2)-963$

C (Vol 3)- 1557, 1614, 1675

Ecological reporting units (ERUs) -

$\mathrm{C}(\mathrm{Vol} 2)-963$ (definition)

Ecological simplification-

C (Vol 2)-963 (definition)

Economic efficiency-

$\mathrm{S}-40$

C (Vol 1)- 20,81

C (Vol 4) - 1723-1724, 1728, 1805, 1835

Economic resiliency (see also: Community resiliency; Resiliency; Social resiliency) -

F- 17, 20

I- 12-13, 30, 35, 96, 124-125, 126 (Figure 42), 127-128, 129 (Figure 43), 134-135, 148, 153-155, 181, Appendix B

S- 24, 129, 131, 138, 140

C (Vol 1)- 11

$\mathrm{C}($ Vol 2) - Appendix 3M

$\mathrm{C}(\mathrm{Vol} 4)-1810-1811$

Economic value (see also: Attitudes, beliefs, and values; Culture/Cultural)_

C (Vol 3) - Chapter 6 Economic Assessment, 1723-1725, 1728, 1734-1735, 1738, 1743 , $1745,1746,1748-1751,1753,1756,1766-1767,1772,1774,1779-1780$, $1782-1783$

Economy-

$\mathrm{F}-8$

$\mathrm{H}-22$

I- $56,76,82,8,86,91,124,127-128,168$

$\mathrm{S}-14-15,18,31,35,41-43,45,55,61,115,129$

C (Vol 1) - 10, 19, Appendix 1A, 83-84, 88, 90-91

C (Vol 3) - 1073, 1511, 1513

C (Vol 4) - Chapter 6 Economic Assessment, 1723-1725, 1728, 1731-1732, 1735, 1737-1738, 1743, 1750, 1753, 1767, 1782, 1806-1808, 1810-1814, 1817, 1833, 1837-1838, Chapter 7 Social Assessment, 1886, 1888-1890, 1895, 1907, 1915, 1929, 1934, 1936, 1939-1941, 1944-1945, 1953, 1956, 1964, 1966

Ecoregion-

$\mathrm{F}-19,27,45$

I- $21,34,174,177$

$\mathrm{S}-58$

C (Vol 1)- 23, 90, 113, 120,313

C (Vol 2)- 374, 376 (Map 3.1), 517, 554, 572, 585, 592, 602, 610, 620, 624, 635, 643, 655, $661,963,965$

$\mathrm{C}(\mathrm{Vol} 3)-1378$

C (Vol 4) - 1819, 1908, 1966, 2029, 2032, 2038 
Ecosystem analysis-

F- 15, Appendix B

I- 142, 148, Appendix A

C ( Vol 2)- 919

$\mathrm{C}($ Vol 3) - 1372, 1651

Ecosystem assessment-

I- 36

C (Vol 1)- 118

C (Vol 4)- 1974, 1976

Ecosystem goods, functions, conditions--

$\mathrm{F}-18,21-22,26$

I- $12,30,32,35,37-39,45,82,127,154$

$\mathrm{S}-24,40$

$\mathrm{C}($ Vol 1) -81

C (Vol 3)- 1375

C (Vol 4) - 1724-1725, 1814, 1816-1817, 1835, 1958

Ecosystem health-

F- 4, 7, 9, Appendix B

$\mathrm{H}-30$

I- $17,27,74-75,138,168,170,178$, Appendix A

S- 22, 93, 134, 138

C (Vol 1) - 10, 12, Appendix 1A, 47-48, 51-53, 62, 65, 73, 78, 81, 137-138

$\mathrm{C}(\mathrm{Vol} 2)-390,406,918,965$

C (Vol 3) - 1107, 1142, 1260, 1512, 1532, 1555, 1608, 1648

C (Vol 4) - 1796, 1817-1818, 1823, 1828, 1836, 1891, 1909, 1957

Ecosystem integrity-

F- 4, 17, 22, 26, 36, 45, Appendix B

I- $12,29-30,34,38,95,134,157,171,187$, Appendix A

$\mathrm{S}-22,101,115$

$\mathrm{C}($ Vol 1)- 11, 19, 23, 66, 78

$\mathrm{C}(\mathrm{Vol} 2)-880,908$

$\mathrm{C}(\mathrm{Vol} 3)-1339$

C ( Vol 4)- 1729

Ecosystem management (see also: Ecosystem principles) -

F- 3-5, 7, 9-11, 13, 15-17, 19, 21-22, 23-37, 45 (definition), Appendix B

I- 11-13, 17-19, 22, 27, 29, 30-33, 37-39, 41, 45, 75, 91, 95, 128, 140, 163, 166-169, $173-175,177-178,180-181,183,185,187$

$\mathrm{S}-11,15,18,22-26,28,37-38,40,46,51-59,61,65-66,92,93,100,111,115,123,142$

$\mathrm{H}-12$

C (Vol 1) - 8, 10-12, 23 (definition), 68-70, 73, 75, 81, 87, 88-90, 92-93, 97, 98

C (Vol 2)- 363, 908, 917, 925, 926, 963, Appendix 3M

C (Vol 3)- 1113, 1142, 1238-1239, 1354, 1356, 1363, 1523, 1528, 1610-1611, 1643-1644, $1646-1648,1654$

C (Vol 4)- 1725, 1745, 1798, 1810, 1814-1833, 1835, 1877, 1879-81, 1890-92, 1897-1899, $1901,1903,1905,1907-1913,1915-1916,1931-1932,1933-1938,1949$, 1951-1952, 1955-1957, 1966-1969, 1971, 1973, 1974, 1975-1977, 1979, 1981-1990 
Ecosystem principles (see also: Ecosystem management)—

F- 5, 9, 36, Appendix B

I- 173, Appendix A

$\mathrm{S}-18,24$

C (Vol 1)- 14

Ecotone-

I- 114

$\mathrm{S}-128$

C (Vol 2)- 480, 964

Ecotypic variation-

C (Vol 2)- 384, 964

Ectomycorrhizae-

$\mathrm{C}(\mathrm{Vol} 3)-1675$

El Niño-

C (Vol 1)- 190, 194, 310, 314

Employment-

$\mathrm{F}-16,24$

$\mathrm{H}-22$

I- 29, 76, 81-82, 124-125, 168, Appendix B

$\mathrm{S}-42-44,49,55-56,129,138$

$\mathrm{C}($ Vol 1) - 83-86

C (Vol 4) - 1732, 1734-1735, 1737, 1739-1744, 1750, 1753, 1756, 1785, 1788-1789, 1793, 1796, 1806-1807, 1810-1813, 1832, 1837-1840, 1888, 1898, 1914, 1933, 1936-1937, 1939-1943, 1945-1951, 1953, 1955-1956, 1964, 2031

Encroachment-

I- $16,114,123,153,164,166,179,184$

$\mathrm{S}-128$

$\mathrm{C}(\mathrm{Vol} 2)-418,433,480,500,502,530,549,556,560,624,644,673,679,685,690,702$, $706,709,716,718,720,722,726,755,783,822,834,849,852,888,894,896$, 964, Appendix 3E

C (Vol 3)- 1332, 1583

C ( Vol 4)- 1924, 1952

Endangered species/Endangered Species Act (see also: Candidate species; Fish, rare and sensitive;

Rare and sensitive species; Threatened or endangered species) -

F- 5, 19, 24, Appendix B

$\mathrm{H}-21,32$

I- $14,16,34,57,75,103,171,182,184$, Appendix A

$\mathrm{S}-22,37,93$

$\mathrm{C}(\mathrm{Vol} 1)-12,73,88$

$\mathrm{C}($ Vol 2)- 390, 392, 908, 922-923

C (Vol 3) - 1073-1074, 1081, 1098, 1141, 1160, 1176, 1201, 1213, 1223, 1241, 1249, 1251 (Map 4.33), 1267, 1271, 1284, 1289, 1298, 1301, 1337, 1359, 1364, 1511, $1519,1543,1568,1572,1595,1581,1644$, Appendix 5E

C (Vol 4) - 1773, 1816-1818, 1820, 1891, 1902, 1905-1906, 1913, 1915, 1919-1920, 1951, 1969,1980 
Endemic species/Endemism -

F- 18,45

I- 14, 57, 75, 78 (Figure 22), 79 (Figure 23), 120, 146, 167, 183

S- 93, 97, 98 (Map 14), 99-100, 139

C (Vol 1) - 24, 67, 73, 76-78, 79 (Map 1.9), 116

C (Vol 2)- 384, 697

C (Vol 3) - 1149, 1241, 1249, 1250 (Map 4.32), 1263, Appendix 4C, 1507-1508, 1519, $1522,1529-1531,1533,1535,1537,1566,1591,1615,1618-1619,1623,1624$ (Map 5.4), 1625 (Map 5.5), 1626, 1628, 1637-1638, 1644, 1646-1647, 1652, 1675, Appendix 5D, Appendix 5H

Energy -

C (Vol 4)- 2028, 2044

$\mathrm{F}-7-8,18$

I- $21,52,59,100,165$

$\mathrm{S}-18,80,131$

C ( Vol 1)- 56, 108, 144-148, 175, 226, 228-229

C (Vol 2) - 364-369, 371-373, 375, 382-383, 386-391, 405-406, 446, 462, 466, 517, 554, $572,585,593,636,644,766,768,771,777,798-799,805-807,815,824,843$, $845,879-880,908-909,917-918,920-921,923,963$, Appendix 3M

C (Vol 3) - 1101, 1141, 1146, 1301, 1365, 1371, 1375, 1512, 1556, 1559, 1562, 1569, 1608,1675

C (Vol 4)- 1878, 1942, 1968, 1989, 2025

Environmental correlate (see: Key environmental correlate)

Environmental initiatives-

I- 60

Equilibria/Equilibrium (see also: Ecological disequilibria/Ecological disequilibrium)_-

C (Vol 1) - 48, 50-51, 57, 62, 205

C (Vol 2) - 368-369, 371-373, 382-383, 386, 389-390, 393, 406, 445-457, 881, 907-911, 917-918, 920-926, 963-964, Appendix 3M

C (Vol 4)- 1738

Equity-

$\mathrm{F}-12$

S- 20, 40-41, 56

C (Vol 1)- 20, 81-82, 84

C (Vol 4) - 1723, 1784, 1816, 1835-1836, 1838

Equivalent Potential Temperature-

C (Vol 1) 310 


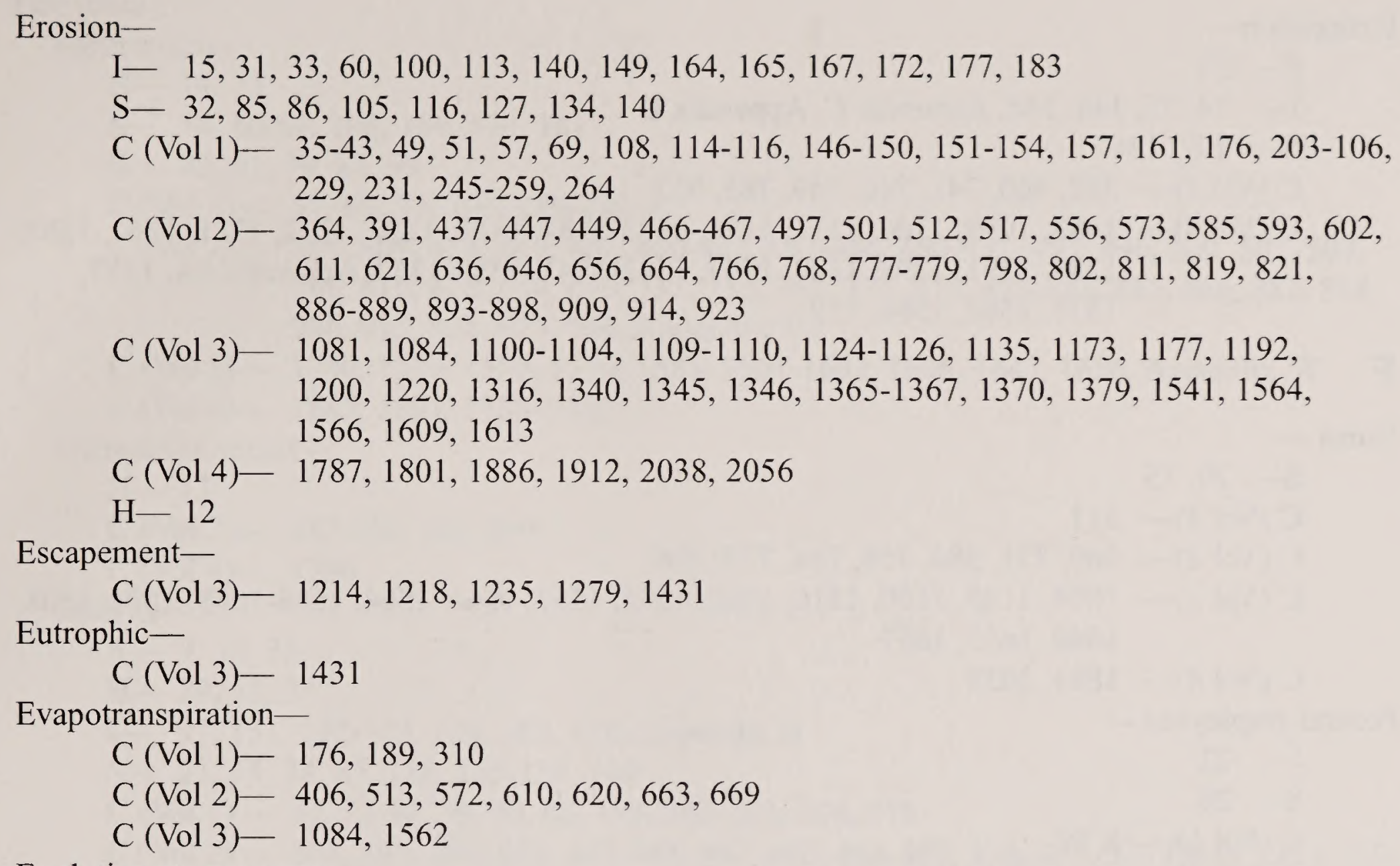

Evolutionary processes-

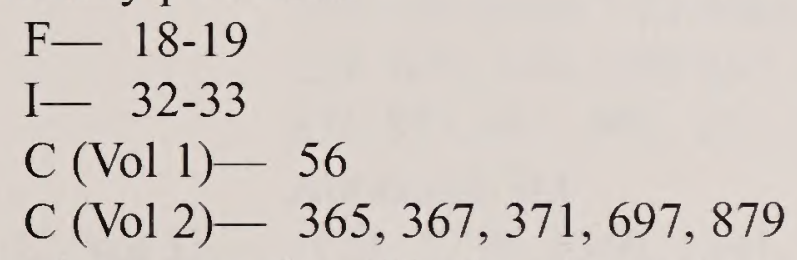

Exotic plants (see also: Weeds, noxious) -

$\mathrm{F}-9$

$\mathrm{I}-60,164$

$\mathrm{S}-82,95,135$

C (Vol 1) - 62

C (Vol 2) - 433-436, 447-448, 459-460, 477, 500, 509, 530, 536, 572, 579, 592, 621, 626, $646,664,670-671,716,764-765,778,782-795,801,834,843,885-886,896$, $900,907,910-911,914,916$

$\mathrm{H}-13,31,33$

Expert panel-

I- Appendix C

C (Vol 1) - 13, 201, 205

C (Vol 3) - 1509, 1516-1519, 1522-1523, 1527, 1529, 1543-1544, 1554, 1581, 1589, 1591, $1608,1615,1628,1648,1658,1676$, Appendix 5A

Extent (geographic)-

C (Vol 4) - 1803, 2023-2029, 2031, 2048, 2059, 2065 
Extirpation-

$\mathrm{F}-32$

I- 14, 75, 144, 166, Appendix C, Appendix D

S- 107,136

C (Vol 2)- 382, 460, 741, 746, 749, 785, 922

C (Vol 3) - 1083, 1099, 1106, 1111, 1176, 1183-1184, 1188, 1192, 1212, 1221, 1261, 1285, $1288,1291,1298,1327,1337,1342-1343,1359-1360$, Appendix 4A, 1537, $1575,1582,1584,1591$

$\mathbf{F}$

Fauna -

$\mathrm{S}-29,75$

C (Vol 1)- 311

C (Vol 2)- 369, 371, 384, 756, 764, 777, 806

C (Vol 3) - 1074, 1149, 1160, 1316, 1318, 1554, 1557, 1561, 1564, 1566-1568, 1591, 1608, $1640,1653,1657$

C (Vol 4)- 1883, 2033

Federal employees -

$$
\begin{aligned}
& \text { I- } 22 \\
& \text { S- } 26 \\
& \text { C (Vol 1)- } 8,92 \\
& \text { C (Vol 4)- } 1949,1953,1974
\end{aligned}
$$

Federal revenue sharing -

$$
\begin{aligned}
& \mathrm{H}-22 \\
& \mathrm{C}(\text { Vol } 1)-82 \\
& \mathrm{C}(\text { Vol } 4)-1949,1952-1953
\end{aligned}
$$

Fire -

crown -

S $-82-83,89$

C (Vol 2)- 398, 448, 488, 492-493, 496, 545 (Map 3.32), 586, 593, 603, 626, 646, 664, $818,866-867,869,873,890,895,897,906$

frequency -

I- 61, 97, 108-110, 115-117, 119

$\mathrm{C}(\mathrm{Vol} 1)-76$

C (Vol 2)- 366, 372, 404, 418-419, 533 (Map 3.23), 572, 592, 663, 767, 775, 805, 822, $856-857,884-885,909,962,964-965$

C (Vol 3) - 1340, 1536-1537, 1601, 1603, Appendix 5F

ground/surface (see also: Underburn) -

$\mathrm{S}-89$

C (Vol 2)- 169

prescribed-

$\mathrm{H}-31$

I- $16,113,116,119,131-132,138,142-143,149,153,184$

S- $123,127,136$

C (Vol 1)- 204

C (Vol 2)- 401, 443, 447, 476-477, 567-568, 600, 610, 635, 822, 846-854, 867, 876, 906, 915-917, 921, 966, Appendix 3K, Appendix 3L

C (Vol 3) - 1340, 1605-1606, Appendix 5F

C (Vol 4)- 1957, 2039, 2043 
Fire (cont.) -

suppression-

$\mathrm{H}-14,33$

I- $14,60-61,108,164,170,181$

$\mathrm{S}-22,51,79,87,99$

$\mathrm{C}($ Vol 1) - 57, 60, 77, 205, 229-230

C (Vol 2)- 364, 404, 418, 449, 462, 480-481, 487-488, 490-491, 493, 496, 500, 505, 507, $568,610,628,644,696,733,753,762,775,817,821-823,855,866,882,885$, 906, 915, 921, 925, 966, Appendix 3M

C (Vol 3) - 1340, 1536, 1559, 1570, 1583, 1601-1602, 1606, 1647, 1656, Appendix 5F

$\mathrm{C}(\mathrm{Vol} 4)-1887,1891,1985-1986$

tolerant/intolerant-

$\mathrm{H}-13$

C (Vol 2)- 887-888, 891, 895

$\mathrm{C}(\mathrm{Vol} 4)-1796$

wildfire-

F- 9, 12, 33

$\mathrm{H}-29,31,33$

I- $97,131,142-143,149,165,179$, Appendix B

S- $21,34,74,87,116,135-135,140$

C (Vol 1) - 49, 53-54, 56, 60, 68, 193, 202-203, 264, 276

C (Vol 2) - 364, 401, 404, 418, 443-444, 447, 460, 466-467, 476, 477, 529 (Map 3.21), 530, 544-546 (Map 3.31-Map 3.33), 553, 560, 567, 573, 586, 593, 597, 603, 611, $621,636,646,650,656,658,664,785,797-798,801,805,845-856,867-874$, 876-877, 901, 906, 911, 916-917, 921, 924, Appendix 3K, Appendix 3L, Appendix 3M

C (Vol 3) - 1084, 1339, 1340, 1341, 1342, 1346, 1365, 1515, 1563, 1578

C (Vol 4)- 1889, 1985, 1989, 2032, 2039, 2043, 2045

Fish-

anadromous (see also: Salmonids, key)-

F- 33, Appendix B

I- $39,117,123,167,174$, Appendix A

S- 9, 101, 104, 107

C (Vol 1) - 5, 10, 12, Appendix 1A, 63, 67, 74, 175, 183

C (Vol 3) - 1083, 1093, 1099, 1124-1125, 1157, 1160, 1164, 1168, 1213, 1218, 1221-1222, $1228,1234,1241,1249,1254-1256,1259-1260,1264,1278-1279,1351,1354$,

C (Vol 4)- 1791 1363, Appendix 4C, Appendix 4G

rare and sensitive-

$\mathrm{C}(\mathrm{Vol} 3)-1267-1337$

resident-

I- 34

C (Vol 3)- 1168

Flood plain-

I- 100

$\mathrm{S}-75,116$

C (Vol 1)- 144, 146-148, 157, 250, 310

C (Vol 2)- 402, 431-432, 585, 593, 636, 768, 823, 965

$\mathrm{C}($ Vol 3) - 1099, 1112, 1118, 1332, 1365 
Flora-

I- Appendix E

S- 29, 75, 95-97

C (Vol 1)- 75, 77, 321

C (Vol 2)- 369, 384, 756, 776, 806, 893, 897

C (Vol 3) - 1519, 1533, 1536, 1538-1540, 1542, 1563, 1566-1568, 1587, 1600, 1637 , $1650-1651,1657$

C (Vol 4)- 1883, 1938, 1943, 2033

Forage-

$\mathrm{H}-1,15,23$

I- 37, 56, 74, 84, 86, 90 (Figure 30), 114, 117, 119-120, 138, 167

$\mathrm{S}-30,42,51,128,133,138$

C (Vol 1) - 10, Appendix 1A, 57, 83-85

C (Vol 2)- 364, 394, 592, 603, 636, 767, 770, 776, 780, 784, 798, 801, 804, 880, 882, 908, Appendix 3M

C (Vol 3) - 1100, 1106, 1184, 1237, 1278, 1289, 1511, 1520, 1536, 1563, 1567, 1573-1574, $1584,1602,1604-1605,1650,1653$, Appendix 5G

C (Vol 4) - 1724, 1767-1770, 1772-1773, 1817, 1821, 1837-1839, 1937, 2041

Forest clusters-

I- 105-109, 111 (Figure 38), 113, 115-117, 161

S- 119, 120 (Map 19), 121-123, 126-127, 141

C (Vol 1) - 267, 269, 273 (Map 2.42), 274

C (Vol 4)- 2029

Forest health-

F- 10, Appendix B

I- $16,178,184$, Appendix A

$\mathrm{S}-9,34,37,59$

C (Vol 1)- 5, 8, 10, Appendix 1A, 55, 89

$\mathrm{C}(\mathrm{Vol} 2)-918$

C (Vol 3) - 1342, 1372, 1654, 1660

C (Vol 4) - 1878, 1889, 1906, 1909-1911, 1975

Forest integrity-

$\mathrm{H}-10$

I- 97, 98 (Figure 33), 109-110, 113, 117, 134

S- 116, 121-122, 125, 127

C (Vol 1)- 62

C (Vol 2)- 413-415, 416 (Map 3.8), 418, 833, 835, 900

Forest land(s) -

I- 44-45, 54, 56, 96-97, 100, 105, 108, 123, 142, 180

$\mathrm{S}-52,56,85,104,115-116,119$

C (Vol 1) - 36-37, 39-43, 56, 90, 143, 151, 245-247, 251-254, 256-257, 259, 264, 269, 274

$\mathrm{C}($ Vol 3) - 1554

C (Vol 4) - 1907, 1914, 1961-1962

Forest plan(s) (see also: Northwest Forest Plan)-

$\mathrm{F}$ - Appendix B

I- 174, Appendix A

C (Vol 4)- 1955, 1972 
Fragmentation (habitat) -

I- $14-15,45,73,103,149,181,183$

$\mathrm{S}-57,76,107,111$

C (Vol 1)- 49, 52, 54, 65

C (Vol 2) - 411, 413, 739, 741, 757-759, 761-762, 806, 820, 901, 903, 916-917

$\mathrm{C}(\mathrm{Vol} 3)-1106,1110-1113,1173,1184,1200,1212,1260,1262,1275,1314,1320,1330$, $1357,1364,1372,1524,1531,1535,1537,1585,1589-1590,1646$

C (Vol 4)- 1897, 1899, 1919

Framework, ecosystem management-

F- 3-5, 7-10, 16-17, 33-34, 36-37, Appendix B

I- $11,13,17,22,33-34,138,181$, Appendix A

$\mathrm{S}-22,133$

C (Vol 1)- 8, 10-11, Appendix 1A, 56, 81, 110, 120, 137, 172, 313-314

$\mathrm{C}(\mathrm{Vol} 2)-373,390,391-393,420,879,908,924$

C (Vol 3) - 1075, 1077, 1121, 1361, 1375, 1377, Appendix 4C, 1615

C (Vol 4)- 1816, 1831, 1835, 1931, 1976, 1982, 2037, 2049

Fringe -

$$
\begin{aligned}
& \mathrm{I}-146 \\
& \mathrm{~S}-139 \\
& \mathrm{C}(\mathrm{Vol} 1)-67 \\
& \mathrm{C}(\mathrm{Vol} 2)-470
\end{aligned}
$$

C (Vol 3) - 1111, 1183, 1185, 1239, 1245, 1247 (Map 4.31), 1248, 1262-1263

Front (climate)-

C (Vol 1)- 310 (definition), 313

Frontal zone -

$\mathrm{C}(\mathrm{Vol} 1)-188,310$

Fungi (see also: Nonvascular plants) -

I- 58,75

$\mathrm{S}-93-95,100$

C (Vol 1)- 73, 75-76, 78

C (Vol 3) - 1507-1508, 1510-1512, 1518, 1529, 1531, 1535-1536, 1539, 1554-1556, 1564-1565, 1589, 1600-1602, 1611, 1613-1614, 1637, 1644, 1648-1649, 1676, Appendix 5A, Appendix 5C, Appendix 5F, Appendix 5G

\section{G}

Gap winds -

C (Vol 1)- 193, 234, 310

General planning model (GPM)-

$\mathrm{F}-4,23-27,31,36$

I- $19-20,27,175$

$\mathrm{S}-24-25$

Geoclimatic -

$\mathrm{S}-67$

C (Vol 1) - 20, 35, 107-108, 114, 116, 118, 119 (Map 2.3), 120, 121 (Map 2.4), 128, 130, $135,138,140,149,151,175,177,212,310$

C (Vol 3)- 1135 
Geographic Information Systems (GIS)-

$$
\begin{aligned}
& \text { F- 29, } 34 \\
& \text { H- } 4,12,34 \\
& \text { I- } 22,75 \\
& \text { S- } 93 \\
& \text { C (Vol 1) - } 8,13,20,73,90-92,95,96-98,129,137,145,149,171,214 \\
& \text { C (Vol 2) - } 398,404,436 \\
& \text { C (Vol 3) - } 1377, \text { Appendix 4C, 1528, 1530, 1651, 1658 } \\
& \text { C (Vol 4)- } 1962,1968,1977,1984,2017-2018,2020-2024,2031,2034-2039,2042-2051, \\
& \quad 2054-2060,2065-2066
\end{aligned}
$$

Geology -

$$
\begin{aligned}
& \mathrm{I}-31-32,43,179 \\
& \mathrm{~S}-67,75 \\
& \mathrm{C}(\operatorname{Vol} 1)-35,108,111-112,114,118,120,122-123,137,145-148,151,175-176,231, \\
& \text { C (Vol 2)- 364, 517,636,656 } \\
& \text { C (Vol 3)- } 1084,1100,1183,1359,1360,1379-1380,1530,1619-1621,1623 \\
& \text { C (Vol 4)- } 1958,2027,2032,2038
\end{aligned}
$$

Geomorphology -

$\mathrm{C}(\mathrm{Vol} 2)-373,388,517,602,880,965-966$

$\mathrm{C}($ Vol 3) - 1100, 1380

Goals (see: Management goals)

Goods and services -

$$
\begin{aligned}
& \text { F- 4, 9, 11-13, 20-22, 26- } 27,35,37,46 \\
& \text { H- 29, } 34 \\
& \text { I- 21-22, 32, 40, 86, 95-96, 127, 138, 148, 154, 168, 174, } 185 \\
& \text { S- 20-21, 23, 26, 129, 142 } \\
& \text { C (Vol 1) - 11, 25 } \\
& \text { C (Vol 3) - } 1375 \\
& \text { C (Vol 4) - 1737, 1744, 1812, 1817, 1832, 1878, 1903, } 1958
\end{aligned}
$$

Grazing (see also: Overgrazing/Heavy grazing; Ranching) livestock-

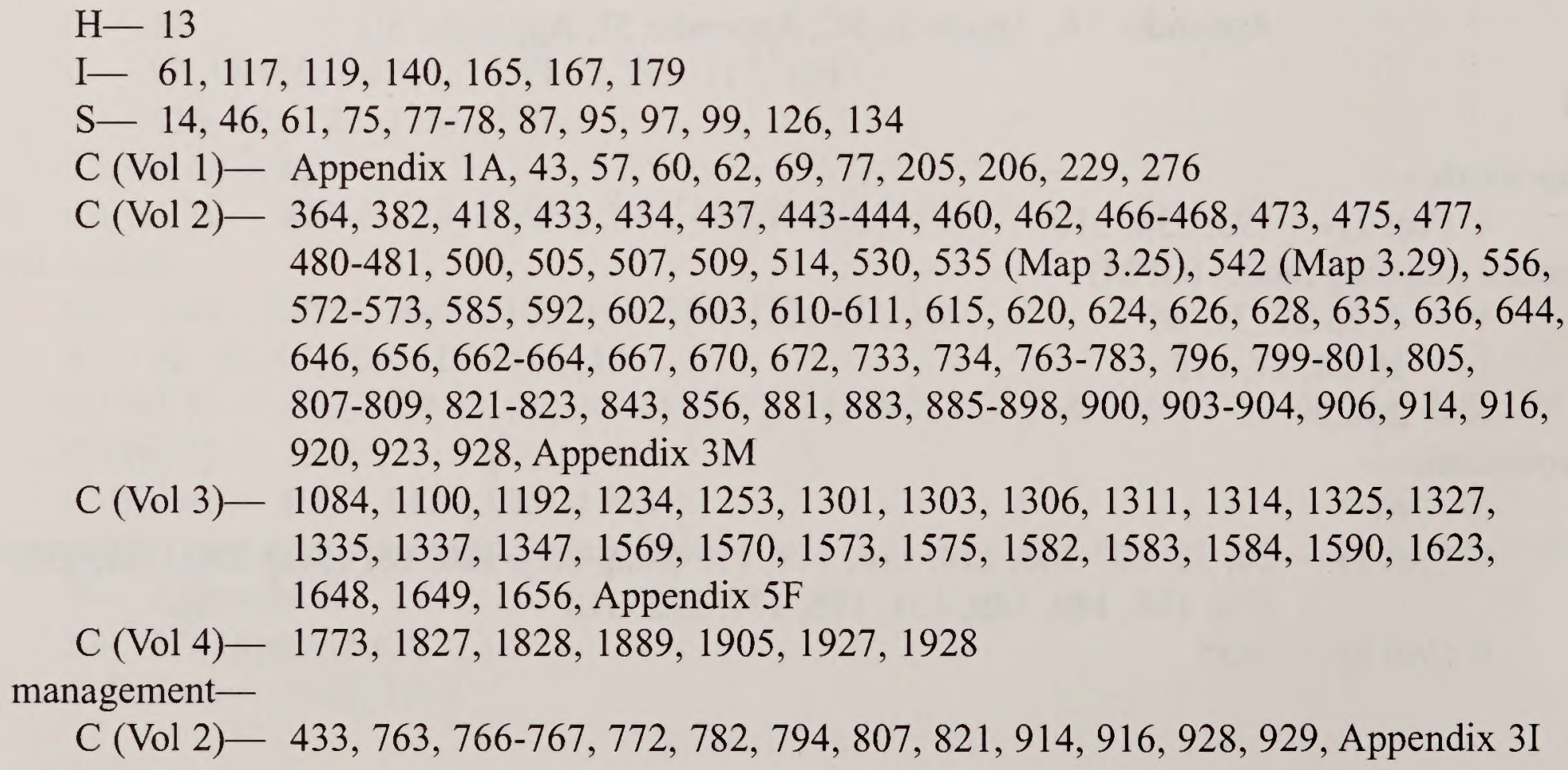


Greater Yellowstone Ecosystem (GYE)-

$\mathrm{C}(\mathrm{Vol} 2)-964,966$

C (Vol 3) - 1574-1575

Greenhouse effect-

C (Vol 1) - 200, 310

Ground water-

$$
\begin{aligned}
& \text { I- } 100 \\
& \text { S- 31, } 116 \\
& \text { C (Vol 1)- 19, 194, 207-208, 229-230 } \\
& \text { C (Vol 2)- } 874,965 \\
& \text { C (Vol 3)- } 1182 \\
& \text { C (Vol 4)- } 1750,1803
\end{aligned}
$$

H

Habitat, aquatic (see: Aquatic habitat)

Habitat, terrestrial (see: Terrestrial habitat)

Habitat type -

C (Vol 2)- 415, 922, 968

C (Vol 3) - Appendix 4C, 1525, 1557

Hatchery/Hatcheries-

$$
\begin{aligned}
& \mathrm{H}-17 \\
& \mathrm{I}-105 \\
& \mathrm{~S}-112 \\
& \mathrm{C}(\mathrm{Vol} 1)-67-68
\end{aligned}
$$$$
\text { C (Vol 3) - 1074, 1083, 1106-1108, 1162, 1168, 1194, 1212-1213, 1218, 1221-1223, 1228, }
$$
$1232,1235-1236,1248,1260,1282,1284-1285,1288,1335,1342,1356,1359$, 1361, Appendix 4C

C (Vol 4) - 1748-1749, 1784, 1808, 2026, 2032

Headwater(s)-

I- 34,115

$\mathrm{S}-46,67,104$

C (Vol 1)- 67, 181, 184, 188

C (Vol 2) - 529 (Map 3.21), 517, 556, 572, 592, 602, 610, 620, 624, 635, 643, 655, 661

C (Vol 3) - 1111-1112, 1121, 1126, 1136, 1150, 1173, 1176-1177, 1182, 1184-1185, $1193-1194,1198,1264,1285,1288,1335,1346,1360,1363,1366$,

Appendix 4D, Appendix 4F, Appendix 4G, 1627

C ( Vol 4)- 1749, 1889

Heavy grazing (see: Overgrazing)

Herbivore-

C (Vol 2) - 367, 447, 764, 766

C (Vol 3) - 1560-1561, 1563, 1567, 1568, 1653, 1654, Appendix 5G 
Hierarchy-

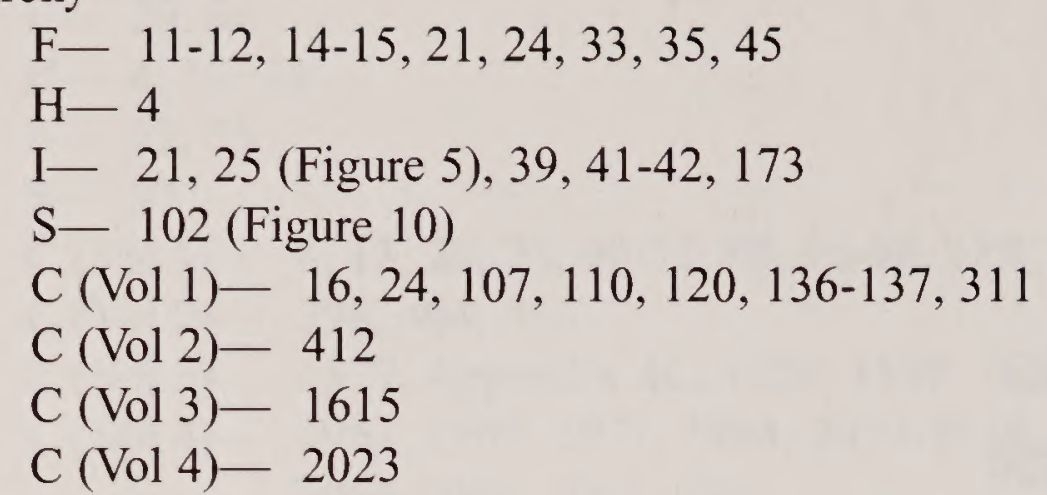

High-quality water-

$$
\begin{aligned}
& \text { I- } 103 \\
& \text { S- } 116 \\
& \text { C (Vol 2)- } 961 \\
& \text { C (Vol 3)- } 1348
\end{aligned}
$$

Holocene -

$$
\begin{aligned}
& \mathrm{C}(\text { Vol } 1)-147,189,311 \\
& \mathrm{C}(\mathrm{Vol} 2)-763-765 \\
& \mathrm{C}(\mathrm{Vol} 3)-1082
\end{aligned}
$$

Home range-

$\mathrm{I}-75$

C (Vol 3) - 1525-1527, 1530, 1590, 1592-1595, 1626-1627, 1646

Hot spot-

$\mathrm{F}-18$

I- $14,75,183$

S- 98 (Map 14), 100

C (Vol 1)- 77-78, 79 (Map 1.9), 116

C (Vol 3) - 1529, 1618-1621, 1623, 1624 (Map 5.4), 1626, 1628, 1644

Humidity C ( Vol 4) - 2028

$\mathrm{C}($ Vol 1) - 188-189, 311, 313

$\mathrm{C}($ Vol 2) - 405, 819

$\mathrm{C}(\mathrm{Vol} 3)-1365$, Appendix 5F

Hybridization-

C (Vol 3) - 1106, 1161, 1184, 1188, 1191, 1193, 1199-1200, 1210-1212, 1238, 1260-1261, $1263,1278,1320,1322,1332,1337,1648$

Hydrography-

C (Vol 1) - 123, 149, 157, 311

C (Vol 3) - 1079 (Figure 4.1), 1085, 1121-1122, 1124, 1136

Hydrologic cycle-

$\mathrm{C}(\mathrm{Vol} 1)-226,228$

C (Vol 2) - 365-366, 391

Hydrologic hierarchy-

$\mathrm{H}-4$

I- 25 (Figure 5)

S- 102 
Hydrologic integrity-

$\mathrm{H}-10-11$

I- 100, 101 (Figure 35), 102 (Figure 36), 103, 108-110, 113-117, 119, 121, 123, 134

$\mathrm{S}-116,121-122,125,127-128$

C (Vol 1) - 20, 43, 44 (Map 1.5), 45 (Map 1.6), 108, 230, 259-260, 264, 267-269, 270

(Map 2.39), 271 (Map 2.40), 274, 276, Appendix 2A

C (Vol 2)- 965

Hydrologic resiliency-

I- 103

C (Vol 1)- 260, 264; 274

Hydrologic unit codes (HUCs) (see also: Hydrologic hierarchy; Scale)—

$\mathrm{H}-4$

I- 96

C (Vol 1) - 136-138 (discussion), 140, 149, 151-152, 157, 259-260, 264, 269, Appendix 2A

C (Vol 2) - 374, 378 (Map 3.3), 379 (Map 3.4), 400, 412, 541 (Map 3.28), 543 (Map 3.30), $736,817,965$ (definition), 967-968

C (Vol 3)- 1523, 1530, 1627

C (Vol 4)- 1964, 2026, 2040-2041, 2043, 2046

Hydrology-

I- 96, Appendix B

$\mathrm{S}-115$

C (Vol 1)- 35, 108, 137, 207, 226, 231

$\mathrm{C}(\mathrm{Vol} 2)-517,768,775,777,785,805$

C (Vol 3) - 1077, 1093, 1099-1100, 1115, 1609

Hyphae-

$\mathrm{C}(\mathrm{Vol} 4)-2026,2032$

C (Vol 3)- 1614, 1676

Hypothesis -

$\mathrm{F}-31,45$

I- 174-175

$\mathrm{C}($ Vol 1 $)-24$

$\mathrm{C}(\mathrm{Vol} 2)-777,800$

C (Vol 3)- 1137, 1173, 1198, 1344, 1530, 1645, 1652

C (Vol 4)- 1725, 1808, 1810, 1910, 1918, 1944

I

Implementation-

F- 3-4, 8, 23-28, 30-31, 33, 36, 46, Appendix B

I- 19-20, 27, 39, 131, 137-138, 149, 163, 173, 179, Appendix A, Appendix D

S- 24-25, 59, 65-66, 133-134

C (Vol 1) - 10, 24, Appendix 1A, 91-93, 97-98, 110

$\mathrm{C}(\mathrm{Vol} 2)-363,487,490,505,514,568,670,868$

$\mathrm{C}($ Vol 3) - 1109, 1348, 1354, 1364, 1372

C (Vol 4) - 1773, 1798, 1816, 1920, 1931, 1972-1973, 1975-1976, 1979-1982, 1988, 1990, 2057,2060 
Increaser-

I- Appendix D

C ( Vol 2)- 965

Indicator species-
C (Vol 1)- 130,138
$\mathrm{C}(\mathrm{Vol} 2)-368,966-967$
C (Vol 3)- 1615

Information gaps (see: Research needs/Information gaps)

Infrastructure-

$$
\begin{aligned}
& \text { F- } 29 \\
& \text { H- } 22 \\
& \text { I- } 84,124,128,131,154,166 \\
& \text { S- } 31,41,43,47,49,57,129,131 \\
& \text { C (Vol 1) - } 81-82,89,96 \\
& \text { C (Vol 3) - } 1377 \\
& \text { C (Vol 4) - } 1750,1766,1788,1814,1825,1831,1835,1837,1889,1892,1938-1939, \\
& \quad 1942-1944,1949,1955,1984-1985,2023,2047
\end{aligned}
$$

Inland Native Fish Strategy (INFISH)-

I- 138,142

C (Vol 1) - 10, Appendix 1A

C (Vol 3) - 1348-1352, 1366, 1369

Insect and disease (as a vegetation disturbance)-

C (Vol 2) - 382, 386, 389, 401-406, 460, 466, 468, 473, 476, 481, 484, 487, 490, 492, 493, 496, 514, 545 (Map 3.32), 553, 563, 580, 581, 586, 590, 592-593, 597, 600, $602,603,605,608,615,626,628,629,635,636,641,643,646,650,655,656$, $658,661,664,670,768,816,818,824,828,875,877,884,902,903,905,924$

Integration-

$$
\begin{aligned}
& \mathrm{F}-3,13,24,29,33 \\
& \mathrm{I}-11,17,19,137 \\
& \mathrm{C}(\mathrm{Vol} 1)-14,62,91,136,175,183 \\
& \mathrm{C}(\mathrm{Vol} 2)-390,445-446 \\
& \mathrm{C}(\mathrm{Vol} 3)-1378,1654 \\
& \mathrm{C}(\text { Vol } 4)-1827,1968,2036-2037
\end{aligned}
$$

Integrity (see: Aquatic integrity; Composite ecological integrity; Forest integrity;

Hydrologic integrity; Landscape integrity; Range integrity) Intergovernmental-

$$
\begin{aligned}
& \mathrm{F}-5 \\
& \mathrm{I}-173 \\
& \mathrm{~S}-26 \\
& \mathrm{C}(\mathrm{Vol} 1)-189,200 \\
& \mathrm{C}(\mathrm{Vol} 4)-1968,1985
\end{aligned}
$$

Intermontane-

$\mathrm{S}-61$

C (Vol 1)- 37-40, 42, 91, 124-127, 143, 247-249, 251, 254, 257, 311

Inversion (temperature)-

C (Vol 1)- 199-200, 234, 311

$\mathrm{C}(\mathrm{Vol} 2)-431$ 


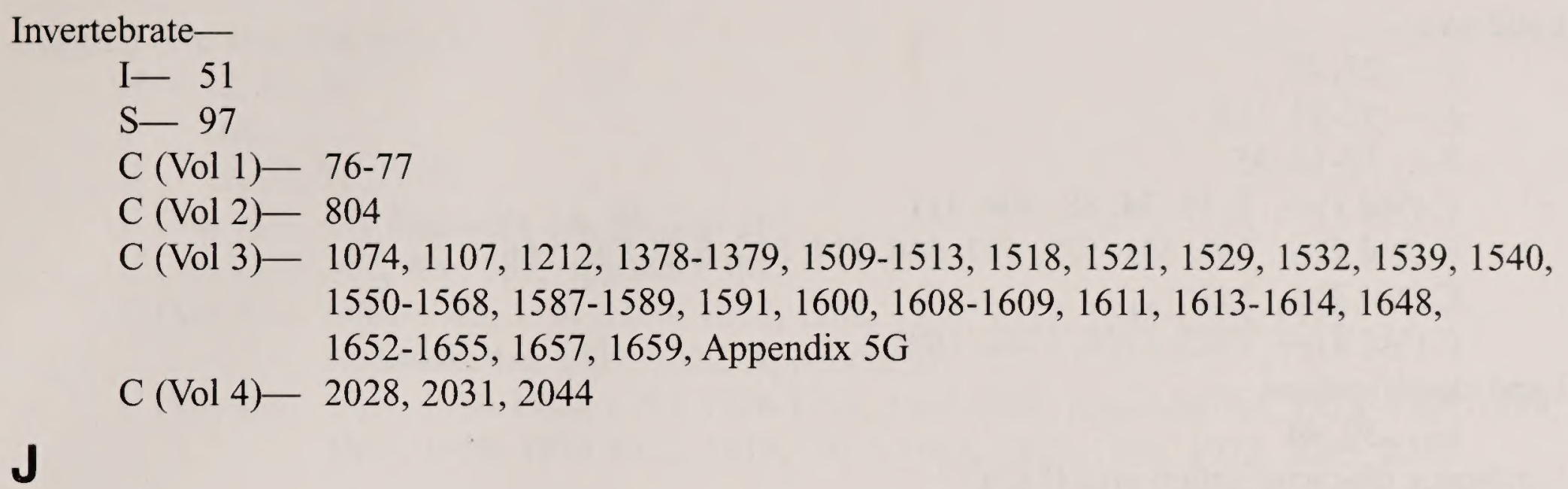

Jet stream-

C (Vol 1)- 188, 194, 311, 313

\section{$\mathbf{K}$}

Key ecological function (KEF)/Ecological function -

$$
\mathrm{F}-18
$$$$
\text { I- } 14,75,148,173
$$$$
\text { C (Vol 1)- } 70
$$$$
\text { C (Vol 2)- } 962
$$$$
\text { C (Vol 3) - 1107, 1176, 1364-1365, 1369, 1371, 1517, 1522-1523, 1527-1528, 1557, 1560, }
$$
1569, 1571, 1608-1609, 1615, 1616 (Map 5.3a), 1617 (Map 5.3b), 1646-1647,

C (Vol 4)- 2044 1657, 1676, Appendix 5G

Key environmental correlate (KEC)/Environmental correlate-

$\mathrm{F}-20$

C (Vol 1) - 20,73

C (Vol 3) - 1517, 1521, 1523, 1527-1528, 1591, 1615, 1643, 1646-1647, 1657-1658, 1676, Appendix 5F

Keystone species-

$$
\begin{aligned}
& \mathrm{S}-99-100 \\
& \mathrm{C}(\text { Vol 1)- } 75 \\
& \mathrm{C}(\text { Vol 3)- } 1608,1614
\end{aligned}
$$

$\mathbf{L}$

Lacustrine-

C (Vol 1)- 38-42, 124-127, 143, 146-148, 178, 181, 248-249, 251, 254-255, 257, 311

C (Vol 3) - 1188, 1190-1191, 1234, 1291, Appendix 5F

Land allocations-

$$
\begin{aligned}
& \text { F- } 18 \\
& \text { I- } 75,163,172 \\
& \text { S- } 100 \\
& \text { C (Vol 1)- } 12,78 \\
& \text { C (Vol 3)- } 1626
\end{aligned}
$$


Land area-

$\mathrm{F}-23,45$

I- $52-54,128$

$\mathrm{S}-13-14,35$

C (Vol 1)- 7, 19, 24, 88, 309, 311

C (Vol 2)- 363, 531, 579, 597, 646, 714, 810, 849, 894, 966

$\mathrm{C}(\mathrm{Vol} 3)-1530$

C (Vol 4)- 1723, 1725, 1782, 1893

Land classifications -

I- 39,44

Landscape characterization area (LCA) -

$\mathrm{C}($ Vol 1) -136

C (Vol 2)- 374, 376 (Map 3.1), 400, 413, 759-762, 965

C (Vol 4)- 2024-2029, 2037

Landscape ecology-

$\mathrm{F}-11,14-15$

$\mathrm{H}-13-14$

I- $17,22,41-42,47,54,60-62,142-143,178,181-182$

$\mathrm{S}-135$

C (Vol 1)- 8, 11, 14, 16, 47, 107-108, 109 (Map 2.1), 110, 118, 120, Appendix 2A

C (Vol 2)— Chapter 3, Landscape Ecology; 363, 373, 392, 405, 441, 966-967

$\mathrm{C}(\mathrm{Vol} 3)-1124,1126,1130,1142$

C (Vol 4) - 1725, 1828, 1832, 1892, 1903, 2021, 2060

Landscape health-

C (Vol 1) - 50, 54, 56

$\mathrm{C}($ Vol 2) - 918, 965 (definition)

Landscape integrity -

$\mathrm{I}-123$

$\mathrm{C}(\mathrm{Vol} 2)-965$ (definition)

Lichens (see also: Nonvascular plants) -

I- 58, 75-76

$\mathrm{S}-93-95$

C (Vol 1)- 73, 75-76, 78

$\mathrm{C}(\mathrm{Vol} 2)-801-802,804-805,875$

C (Vol 3) - 1507-1508, 1510-1511, 1518-1520, 1529, 1531, 1536-1537, 1541, 1565, 1578, 1600-1607, 1610-1611, 1613, 1637, 1644, 1648-1651, Appendix 5A,

Lineage (geographic) Appendix 5F, Appendix 5G

C (Vol 4)- 2047, 2049-2050, 2066

Lithologic-

C (Vol 1)- 37, 115-116, 117 (Map 2.2), 157, 209-210, 246-247, 264

$\mathrm{C}(\mathrm{Vol} 3)-1379$

Loess-

I- 48

$\mathrm{S}-13,68$

C (Vol 1) - 18, 37-38, 40, 115, 123-124, 126, 143, 177, 181, 183-184, 211, 246, 248-250, $254,309,311$

$\mathrm{C}(\mathrm{Vol} 2)-515,672$

C (Vol 3) - 1082, 1127, Appendix 4A 
Logging (see also: Timber)-

$$
\begin{aligned}
& \text { H- 12, 14, } 29 \\
& \text { I- } 168 \\
& \text { S- 22, 32, 34, 55-56 } \\
& \text { C (Vol 1)- 10, Appendix 1A, 98, 204-205 } \\
& \text { C (Vol 2) - } 382,496,768, \text { Appendix 3M } \\
& \text { C (Vol 3)- } 1084-1085,1101-1103,1282,1288,1330,1337,1345-1346,1373, \\
& \text { Appendix 4A, 1601, 1622, Appendix 5F } \\
& \text { C (Vol 4)- } 1787,1789-1790,1792,1796-1799,1807-1808, \text { Appendix 6A, 1878, 1888-1889, } \\
& \quad 1891,1906,1910-1912,1914,1917,1933,1951,1966,1973,2055,2058
\end{aligned}
$$

\section{M}

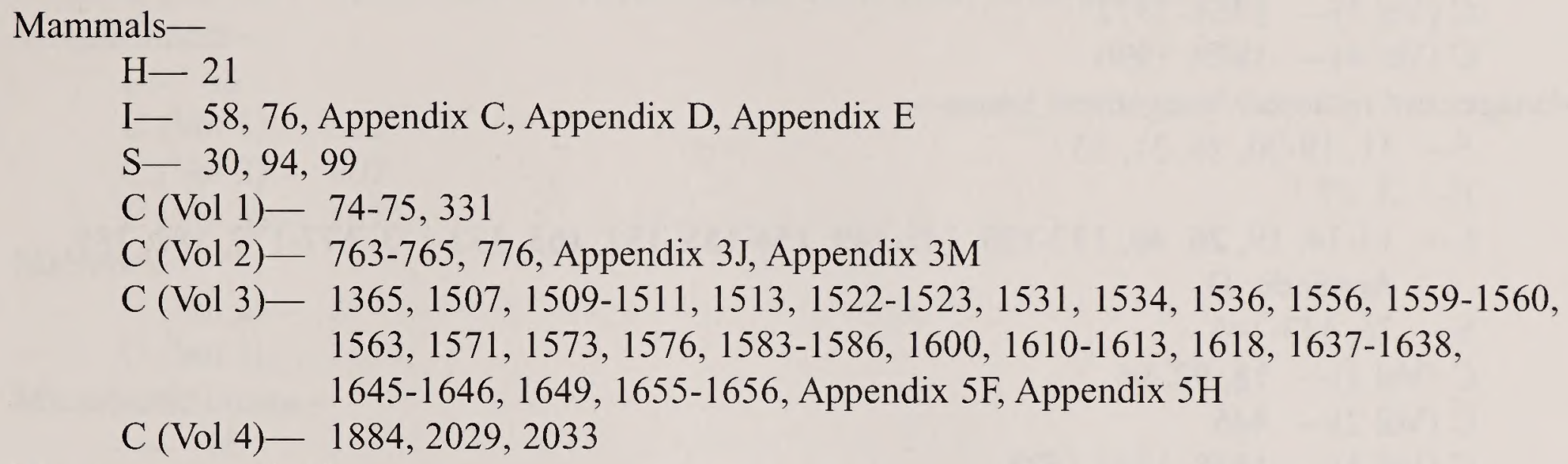

Management activities -

$\mathrm{F}-11,16,18,24,31-32,34$, Appendix B

I- $11,13,24,29,32-33,39,73,75-76,103,105,115,131,140,142,149,165,168,170$, 173-175, 185, Appendix A

$\mathrm{S}-26,40,67,93,95,134-135$

C (Vol 1) - 18, 73, 75, 81, 114, 175, 201, 203-205, 207, 226, 228, 230, 264, 269, 274, 276

C (Vol 2) - 466, 799, 908, 923, Appendix 3M

C (Vol 3) - 1083, 1100, 1102, 1124, 1268, 1330, 1347-1348, 1351, 1356-1357, 1360, $1363-1365,1367,1370-1371,1373-1375,1508,1528-1529,1535,1558,1564$, $1567-1568,1570-1571,1581,1590-1591,1601,1608,1626,1643-1646$, $1651-1652,1654-1655,1657$

C (Vol 4) - 1724, 1787, 1798, 1824, 1835, 1879-1880, 1920, 1953, 1960, 2042, 2052

Management area-

$$
\begin{aligned}
& \text { F- } 13 \\
& \text { S }-26 \\
& \text { C (Vol 1)- } 97 \\
& \text { C (Vol 2)- } 411 \\
& \text { C (Vol 3)- } 1130,1132,1365,1371 \\
& C(\text { Vol 4)- 2032, 2038, 2051, 2052, 2055, } 2057
\end{aligned}
$$

Management class-

C (Vol 2) - 396, 397 (Map 3.7), 398, 420, 432, 441-442, 829, 846, 880, 907, Appendix $3 \mathrm{H}$

C (Vol 3) - 1130, 1131 (Map 4.6), 1133-1134, 1348

C (Vol 4) - 2043, 2051, 2054 
Management direction-

F- 7, 22, 24, 26, 36, 46, Appendix B

I- 19, 95, 145, 157, 171, Appendix A

$\mathrm{S}-133,138$

C (Vol 1) - 10, 24, Appendix 1A

C (Vol 4)- 1988, 2051-2052

Management goals-

$\mathrm{F}-5,15-18,24-26,33$

I- $19-20,27,29,40,173-174,185$

$\mathrm{S}-23-25,142$

C (Vol 1)- 11, 19

C ( Vol 2)- 390, 925

C (Vol 3)- 1364, 1372

C (Vol 4)- 1829, 1990

Management options/Management issues-

$\mathrm{F}-11,19-20,26,31,33$

$\mathrm{H}-3,27$

I- $13-14,19,26,40,137-138,142-149,154-155,157,163,172-173,177-179,182,185$, Appendix D

S- 26, 133-143

C (Vol 1)- 18, 92, 96

$\mathrm{C}(\mathrm{Vol} 2)-446$

C (Vol 3)- 1378, 1522-1523

C (Vol 4)— 1897, 1909, 1917, 1921, 1973-1974, 1980, 1988

Management prescription-

$\mathrm{H}-12$

$\mathrm{I}-40,113-114$

S- 77, 127-128

C (Vol 1)-86, 110, 206

C (Vol 2)- 363, 441-442, 444-445, 447-448, 462, 493, 514, 670, 753, 781, 818, 820, 849, 914, Appendix 3M

C (Vol 3)- 1356

C (Vol 4)- 1796, 1829, 1840

Management regions-

C (Vol 1)- 48, 96

C (Vol 2)- 396, 473, 476, 815, 834, 847-848, 850-851, 853-854, 862, 869, 966

C (Vol 4)- 2028, 2037, 2051

Management scenario(s) -

$\mathrm{F}-33$

C (Vol 1)- 48, 62

C (Vol 2)- 384, 390, 394, 405, 441, 445-446, 448, 471-477, 845-846, 849, 852, 866, $867-869,873,876-877,880,883,906-911,914-917,962$, Appendix $3 \mathrm{H}$

C (Vol 3)- 1221, 1517, 1676

C (Vol 4) - 1827-1829, 2033, 2037, 2039, 2042, 2045, 2051, 2054-2055

Manuscript (geographic) -

C (Vol 1)- 96

C (Vol 4) - 2018, 2036, 2066 


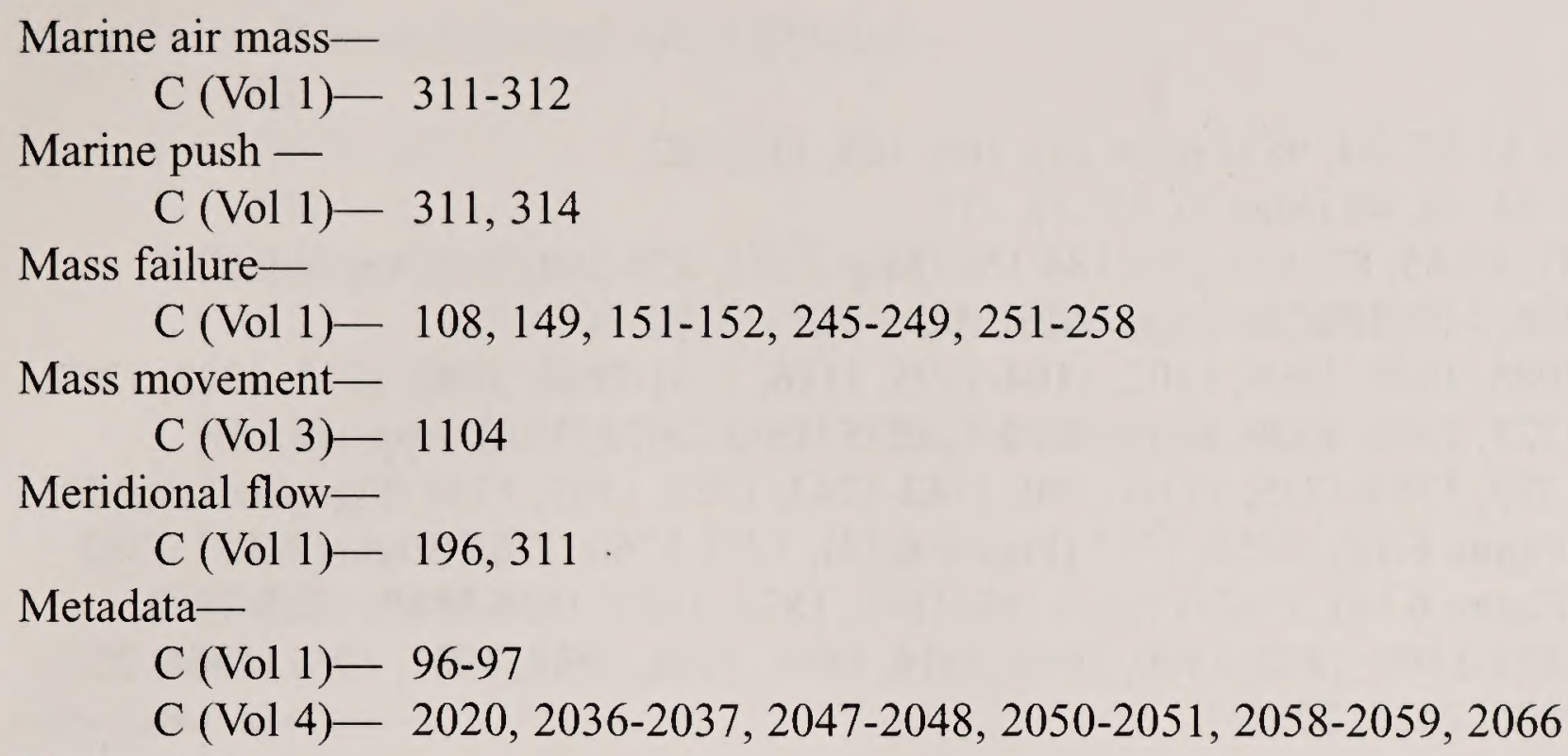

Metasediment-

$$
\begin{aligned}
& \mathrm{I}-48 \\
& \mathrm{C}(\mathrm{Vol} 1)-312 \\
& \mathrm{C}(\mathrm{Vol} 2)-602 \\
& \mathrm{C}(\mathrm{Vol} 3)-1077
\end{aligned}
$$

Microbes-

$$
\begin{aligned}
& \mathrm{C}(\mathrm{Vol} 2)-874 \\
& \mathrm{C}(\mathrm{Vol} 3)-1074
\end{aligned}
$$

Microbiotic crusts-

$$
\begin{aligned}
& \mathrm{I}-76 \\
& \mathrm{~S}-93,95 \\
& \mathrm{C}(\mathrm{Vol} 1)-75 \\
& \mathrm{C}(\mathrm{Vol} 2)-433-434,798,801-802,804-805,889,893-894,898 \\
& \text { C (Vol 3)- } 1537,1644,1648-1649
\end{aligned}
$$

Microclimate-

$$
\begin{aligned}
& \mathrm{C}(\mathrm{Vol} 2)-530 \\
& \mathrm{C}(\mathrm{Vol} 3)-1370,1541,1562-1563,1651
\end{aligned}
$$

Microrelief-

$$
\text { C (Vol 1)- 229, } 312
$$

Migration corridor(s) -

S- 104, 112

C (Vol 1)- 67

C (Vol 3)- 1098, 1147, 1157, 1164, 1168, 1171-1172, 1212, 1214, 1220, 1223-1224, 1255, 1264, 1363, Appendix 4C

Minerals-

$$
\begin{aligned}
& \mathrm{F}-22 \\
& \text { I- } 35,37,43,84 \\
& \mathrm{~S}-49,62 \\
& \text { C (Vol 1)- 20, 81, 85, 116, 118, 176, 179, 184-185, } 186 \text { (Map 2.26), 204, } 247 \\
& \text { C (Vol 3) - 1104, 1188, 1192, 1238, } 1650 \\
& \text { C (Vol 4) - 1723-1724, 1738, 1745, 1750-1751, 1753, } 1754 \text { (Figure 6.11), } 1755 \text { (Figure } \\
& \text { 6.12), 1756, } 1757 \text { (Figure 6.14), 1758-1760, } 1762 \text { (Figure 6.16), 1763-1764, } \\
& \text { 1766, 1799, 1817-1818, 1833, 1835, 1839, Appendix 6A, 1886, 1905, 1928, } \\
& \text { 1940, 1944, 1952, 2021-2022, 2027, 2032, } 2038
\end{aligned}
$$


Mining

$\mathrm{H}-22$

I- $11,21,43,81-82,84,88$ (Figure 28), 100, 103, 115, 127

S- 31-32, 41-44, 46, 48 (Map 6), 49, 57, 116

C (Vol 1) - 43, 82-85, 87, 115-116, 184-186 (Map 2.26), 259-260, 269, Appèndix 2A

$\mathrm{C}(\mathrm{Vol} 2)-383,449,480,764$, Appendix 3M

C (Vol 3) - 1085, 1089, 1095, 1102, 1104-1105, 1116, 1161-1162, 1192, 1212, 1222, 1307 , 1325, 1361, 1370, Appendix 4A, 1575, 1583, 1620, 1650, Appendix 5F

C ( Vol 4) - 1732, 1734-1735, 1738-1740, 1742-1743, 1750, 1753, 1754 (Figure 6.11), 1755 (Figure 6.12), 1756, 1757 (Figure 6.14), 1758-1760, 1761 (Figure 6.15), 1762 (Figure 6.16), 1763-1766, 1799, 1801, 1820, 1833, 1836-1839, 1885-1886, $1888-1889,1892,1900,1906,1914,1940,1946,1948,1951,1957,1966,2022$, $2027,2032,2053$

Mixing height-

C (Vol 1)- 198, 312

Moist forest potential vegetation group (PVG) (see: Potential vegetation group, moist forest)

Monitoring-

F- 3-4, 9, 11-13, 23-27, 31, 34, 36-37, 46, Appendix B

$\mathrm{H}-12$

I- 13, 19-20, 27, 142, 166, 171, 173-175, 179-180, Appendix A

S- 24-26, 29, 65-66, 97, 101

C (Vol 1)- 23-24, Appendix 1A, 53, 64, 71, 73, 76, 91-92, 118, 172

$\mathrm{C}(\mathrm{Vol} 2)-385,805,874-875,877,886,920,963$

C (Vol 3) - 1083, 1088, 1115-1116, 1285, 1337, 1375-1378, 1380, 1508, 1512, 1522, 1530 , $1643,1648-1556$

C (Vol 4) - 1772-1773, 1883, 1931, 1956, 1972-1973, 1975-1977, 1981, 1984-1985, 1990, 2024,2056

Mycorrhizae-

C (Vol 3)- 1539, 1611

$\mathbf{N}$

Native species-

$\mathrm{F}-18-20$

$\mathrm{H}-16$

I- $11-12,30,32-35,45,96-97,103,116,169$

S- 24, 76-77, 79, 94, 111, 115-116

C (Vol 1)- 48, 51, 53, 65-66, 68

C (Vol 2) - 383, 385, 387, 449, 459, 473, 511, 556, 778, 784, 799, 801, 873, 875, 887-892, 895-897, 908, 925-926, 963, 968, Appendix 3M

C (Vol 3) - 1106-1107, 1109, 1143, 1146-1147, 1150-1151, 1154-1155, 1157, 1159-1160, $1184,1221,1238-1239,1241,1242$ (Map 4.28), 1249, 1259, 1262, 1264, 1267, 1293, 1339, 1347, 1356-1357, 1361, 1363, 1373, Appendix 4A, Appendix 4C, $1522,1555,1559,1564,1566,1652-1653$

C (Vol 4)- 1749, 1906 
Natural areas/Research Natural Areas (RNAs) -

$\mathrm{F}-18$

I- $75-76,167$

$\mathrm{S}-100$

$\mathrm{C}(\mathrm{Vol} \mathrm{1})-78,97$

C (Vol 3) - 1107, 1507-1508, 1529-1531, 1615, 1618, 1620-1623, 1626-1627, 1644,1646

C ( Vol 4) - 1778-1779, 1820, 1913, 1915, 2028, 2052, 2054, 2057

Nitrogen-fixing-

$\mathrm{C}(\mathrm{Vol} 1)-205$

C (Vol 2)- 802

C (Vol 3)- 1537, 1541, 1601, 1610

Non-native species-

$\mathrm{F}-18-20$

I- $11-12,30,33-35,45,96-97,103$

$\mathrm{S}-22,24,112,115-116$

C ( Vol 1)- 67

$\mathrm{C}(\mathrm{Vol} 2)-963,968$

C (Vol 3)- 1074, 1105-1107, 1142, 1157, 1159-1160, 1193, 1211, 1221, 1261, 1263-1264, $1306-1307,1311,1316,1318,1322,1372$

Nonpoint source pollution-

C (Vol 3)- 1087, Appendix 4A

Nonvascular plants (see also: Bryophytes; Fungi; Lichens)

$\mathrm{S}-94$

C (Vol 1)- 74

$\mathrm{C}(\mathrm{Vol} 2)-776$

C (Vol 3)- 1534, 1538, Appendix 5G

Northwest Forest Plan (NWFP) -

$\mathrm{F}-15$

$\mathrm{I}-142,163,172$

C (Vol 1) - 10, 12, Appendix 1A, 92, 96, 172

$\mathrm{C}($ Vol 3) -1536

C (Vol 4)- 1891, 1974, 2017, 2032, 2047

Nutrient cycle (see also: Carbon-nutrient cycle) -

I- $31,38,170$

$\mathrm{C}(\mathrm{Vol} 1)-206$

$\mathrm{C}(\mathrm{Vol} 2)-365$

$\mathrm{C}($ Vol 3) -1101

O

Old forest-

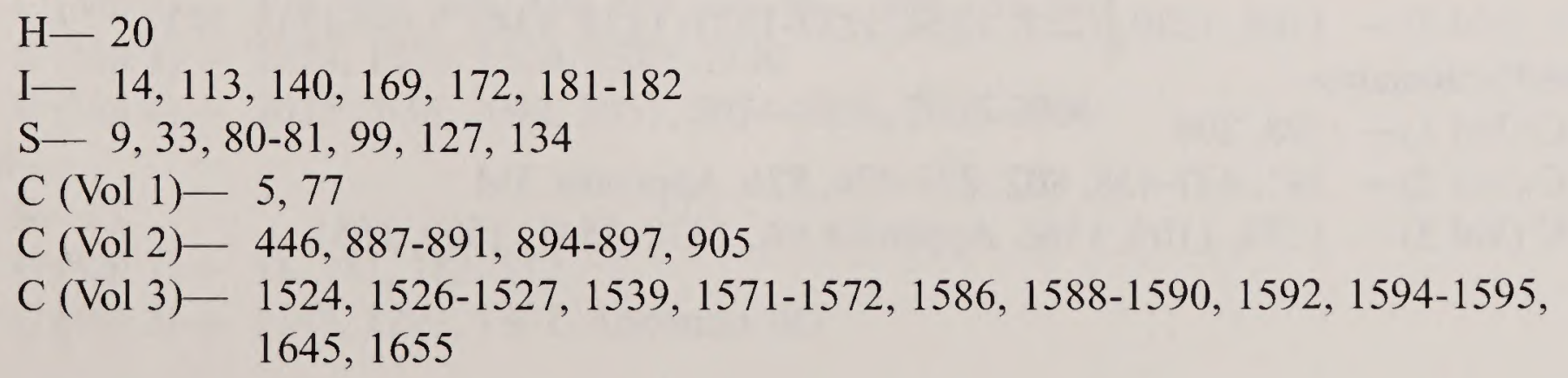


Omnivore-

$$
\text { C (Vol 3)- } 1571
$$

Overfishing-

$\mathrm{S}-46$

C (Vol 3) - 1235, 1285, Appendix 4D

$\mathrm{C}(\mathrm{Vol} 4)-1889,1920$

Overgrazing/Heavy grazing (see also: Grazing) -

$\mathrm{H}-14$

I- $16,114,123,184$

$\mathrm{S}-33,49,51,83,87,97,128$

C (Vol 1)- 77

C ( Vol 2)- 784, 805, 964, 966

C (Vol 3) - 1100, 1301, 1306-1307, 1316, 1322, 1332, 1565-1566, 1621

$\mathrm{C}(\mathrm{Vol} 4)-1887,1920$

Overstory-

$\mathrm{S}-76,80-81,89,123$

C (Vol 2)- 401, 403, 443, 460, 481, 549, 563, 579-580, 586, 588, 597, 605, 615, 628, 638, $641,646,650,816-819,821,823,828,883,889,891-892$

Ozone-

C (Vol 3)- 1568, 1601, 1604, Appendix 5F

$$
\begin{aligned}
& \mathrm{F}-19 \\
& \mathrm{C}(\mathrm{Vol} 1)-311 \\
& \mathrm{C}(\mathrm{Vol} 2)-873-875
\end{aligned}
$$

$\mathbf{P}$

PACFISH (Interim strategies for managing anadromous fish-producing watersheds in eastern

Oregon and Washington, Idaho, and portions of California) -

F- Appendix B

I- $138,142,163,172$, Appendix A

C (Vol 1)- 10, Appendix 1A

C (Vol 3) - 1348-1352, 1355, 1364, 1366, 1369

C (Vol 4)- 1829, 1969, 2026

Pacific air mass-

C (Vol 1)- 188, 312

Pacific North American (PNA) index-

C (Vol 1)- 190, 312

Paleoclimate-

C (Vol 1)- 196, 312

Paleoecological -

Parr-

C (Vol 2)- 365, 383, 386, 401, 433, 961, 966, Appendix 3M

C (Vol 3) - 1109, 1220, 1222, 1224, 1232-1233, 1235, 1342, 1344-1345, 1431

Particles/Particulates-

C (Vol 1)- 198, 204

C (Vol 2) - 391, 437-438, 802, 873-874, 876, Appendix 3M

C (Vol 3) - 1105, 1109, 1366, Appendix 4A, 1538, 1541, 1609, 1651 
Pathogen-

I-61-62, 119, 167

$\mathrm{S}-97$

C (Vol 1) - 57, 77, 111, 204-205, 230

C (Vol 2) - 370, 382-383, 386, 402, 449, 460, 476, 491, 643, 845, 877, 880-881, 887, 902-903, 905-906, 924, 963

C (Vol 3) - 1087, 1340, 1562, 1568, 1613, 1657, Appendix 5F

C (Vol 4)- 1828, 2042, 2051

Payments in lieu of taxes (PILT) -

C (Vol 4)- 1831, 1952-1954

Pedogenic-

C (Vol 2)- 368-369, 372, 382, 386, 390, 966, Appendix 3M

Percolation-

$\mathrm{I}-100$

$\mathrm{S}-116$

C (Vol 1)- 203, 229-230

Perennial-

C (Vol 2)- 438, 965

$\mathrm{I}-21,165$

$\mathrm{S}-79$

C (Vol 1) - 229-230, 322, 324, 326

C (Vol 2)- 387, 403, 406, 432, 438, 440, 459, 477, 497, 500, 502, 504-505, 516, 558 (Map 3.35), 559 (Map 3.36), 572, 574 (Map 3.37), 581, 611, 621, 624, 646, 672, 766-768, 776, 779, 781, 795-800, 807, 809, 821-823, 893, 909, 911, Appendix 3E, Appendix 3J, Appendix 3M

Periglacial-

C (Vol 3) - 1085, 1191, 1213, 1314, 1327, 1366, 1538, 1540, 1565-1566

C (Vol 1) - 40, 42, 125-126, 254, 259-258, 312

C (Vol 2)- 610, 620, 663

C (Vol 3)- 1651

Phenotype-

C (Vol 2)- 367, 697

C (Vol 3) - 1237, 1291, 1360, 1372, 1431

Physiognomic types/Physiognomic type groups (see: Vegetation classifications)

Physiography-

C (Vol 1)- 115, 118, 176, 179, 183, 312

C (Vol 3)- 1081-1082, 1136, 1376

C (Vol 4)- 1944

Pixel (geographic)-

C (Vol 1)- 128, 130, 136-137

C (Vol 2) - 374, 400, 408, 410, 470, 696-697, 699, 734, 962

C (Vol 3)- 1254, 1350, 1524, 1527, 1530

C (Vol 4) - 2035-2036, 2042, 2051, 2054-2055, 2065-2066

Pluvial-

$\mathrm{S}-13$

$\mathrm{C}(\mathrm{Vol} 1)-18,181,183,312$

C (Vol 3) - 1149, 1298, 1307, Appendix 4G 
Point source pollution-

$\mathrm{C}(\mathrm{Vol} 3)-1087$

Polar jet stream (also see: Jet stream) -

$\mathrm{C}($ Vol 1) - 188, 313

Population density-

I- 13, 31, 53, 128, 129 (Figure 43), 131, 135, 137, 153-156, Appendix B

$\mathrm{S}-14,35,131,133$

C (Vol 1)- 19,88

C (Vol 3) - 1570

C (Vol 4)- 1730-1731, 1897, 1929, 1958

Population, human-

$\mathrm{F}-23$

I- $13,21,31-32,43,53,125,127,135,180$

$\mathrm{S}-35,41,47,52,129$

C (Vol 1)- 82, 84-85, 88

C (Vol 2)- 371-372, 923

$\mathrm{C}(\mathrm{Vol} 3)-1085,1354$

C (Vol 4) - 1735-1738, 1743, 1801, 1836, 1839, 1880, 1893-1900, 2031-2032

Population viability (see: Viable/Viable population)

Population viability analysis/Population viability assessments (PVA) -

I- 166

C (Vol 3)- 1344, 1676

Potential natural vegetation-

C (Vol 1)- 118, 122-123, 313

C (Vol 3)-Appendix 4A

C (Vol 4)-2029, 2033

Potential vegetation group (PVG) -

agricultural potential vegetation group (PVG) -

$\mathrm{C}(\mathrm{Vol} 2)-418,419,459,466,500,511,636,656,841$

alpine potential vegetation group (PVG) -

$\mathrm{I}-51$

C (Vol 2) - 456, 458-459, 465, 479, 483, 511, 513, 516, 547, 561, 578, 587, 594, 604, $616,622,627,637,647,657,665,886,910,912-913,916$, Appendix 3A, Appendix $3 \mathrm{H}$

cold forest potential vegetation group (PVG) -

$\mathrm{C}(\mathrm{Vol} 1)-57$

C (Vol 2) - 460, 467-468, 492-494, 496, 514, 567, 597-598, 610, 626, 629, 635-636, 643, $646,650-651,846-847,882-885,900,910,915,921$

cool shrub potential vegetation group (PVG)-

I- 121

C (Vol 1)- 60

C (Vol 2)- 466, 479, 504-506, 549, 562, 579, 621, 624, 643, 667, 885, 910-911

dry forest potential vegetation group (PVG)-

C (Vol 1)- 61-62

C (Vol 2) - 387, 467-468, 481, 484, 487-488, 515, 530-531, 545 (Map 3.32), 549, 563, 567, $579,586,593,597,602-603,605,610,615,628-629,638,644,646,650,656$, $658,849,851,881,883-884,898,900,910,914-915,921$ 
Potential vegetation group (PVG) (cont.)-

dry grass potential vegetation group (PVG) -

$\mathrm{I}-123$

C (Vol 1) -60

C (Vol 2) - 466, 479, 496-497, 499, 500-502, 504, 515, 531, 560, 562, 579, 585, 626, 656, 795,885

dry shrub potential vegetation group (PVG) -

C (Vol 2) - 387, 466, 473, 500-502, 504, 548, 560, 579, 615, 621, 628, 664, 795, 849-850, $852,854,868,885$

moist forest potential vegetation group (PVG)-

C (Vol 1)- 57

C (Vol 2)- 460, 467-468, 488, 490-492, 496, 514-515, 553, 567, 573, 588, 593, 597, 605, $608,638,643,650,658,661,833,846,848,852-853,882,884,898,910,915$

riparian herb potential vegetation group $(\mathrm{PVG})-$

$\mathrm{C}(\mathrm{Vol} 2)-459,479$

riparian shrub potential vegetation group (PVG) -

$\mathrm{C}(\mathrm{Vol} 2)-466,507-508,885$

riparian woodland potential vegetation group $(\mathrm{PVG})$

$\mathrm{C}(\mathrm{Vol} 2)-508-509,628,886$

road potential vegetation group (PVG) -

$\mathrm{C}(\mathrm{Vol} 2)-449,480,512,516,886$

rock potential vegetation group (PVG) -

C ( Vol 2) - 480, 511

urban potential vegetation group $(\mathrm{PVG})$

$\mathrm{C}(\mathrm{Vol} 2)-512$

water potential vegetation group $(\mathrm{PVG})-$

C (Vol 2) - 479, 511

woodland potential vegetation group (PVG) -

C (Vol 2) - 418, 480-481, 508, 514, 515, 884, 910, 914

Potential vegetation type (PVT) (see: Vegetation classifications)

Prescribed fire (see: Fire, prescribed)

Prescription models (see: Management prescription)

Pressure level-

C ( Vol 1)-

$189,310,313-314$

Pronghorn antelope-

I- Appendix C, Appendix E

S- 49

C (Vol 1)- 332

C (Vol 3) - 1574, 1627, 1630 (Map 5.7), 1631 (Map 5.8), 1650

C (Vol 4)- 1887, 1929

Proper functioning condition-

I- 121

$\mathrm{C}(\mathrm{Vol} 2)-768-769,921$

$\mathrm{C}(\mathrm{Vol} 3)-1371$ 


\section{Province-}

ecological/geoclimatic-

$$
\mathrm{F}-14
$$

I- 42

C (Vol 1) - 113, 118, 120, 122-127, 137, 149, 151, 172, 175, 183, 201-202, 313

$\mathrm{C}(\mathrm{Vol} 2)-448$

C (Vol 3) - 1519, 1537, 1542, 1591

C (Vol 4)- 2027, 2029

ichthyological-

C (Vol 3) - 1149-1150, Appendix 4A

Province advisory committee (PAC) -

$$
\mathrm{S}-26
$$

C (Vol 1)- 92

C (Vol 4)- 1974-1975

Public participation-

F- 3, 5, 24, Appendix B

I- 27, 91, Appendix A

$\mathrm{S}-11,24,26,28,59$

C (Vol 1) - 13, 20, Appendix 1A, 91-93

C (Vol 4) - 1881, 1917, 1919-1920, 1971-1977, 1988

Public role (see: Role of the public)

\section{Q}

Qualitative-

$$
\begin{aligned}
& \mathrm{F}-20 \\
& \mathrm{I}-36 \\
& \mathrm{C}(\mathrm{Vol} 2)-398,446,806 \\
& \mathrm{C}(\operatorname{Vol} 3)-1356,1530,1654 \\
& \mathrm{C}(\operatorname{Vol} 4)-1983,2065
\end{aligned}
$$

Quality of life -

$$
\mathrm{F}-20
$$

I- $86,127,145,168$

S- 52, 56, 131, 139

C (Vol 1)- 83, 90

C (Vol 4) - 1737, 1807, 1812-1813, 1832, 1837, 1879, 1881, 1898, 1906, 1930, 1936, 1939, 1942, 1949, 1952, 1957, 1964, 1966, 1968

Quantitative-

$$
\begin{aligned}
& \text { F- } 20 \\
& \text { I- } 24 \\
& \text { C (Vol 1)- } 135 \\
& \text { C (Vol 2) - } 398,437,446 \\
& \text { C (Vol 3)- } 1134-1136,1161,1163,1365,1524,1530,1654,1657,1676 \\
& \text { C (Vol 4)- } 1758,1762,2065
\end{aligned}
$$

Quaternary-

C (Vol 1)- 312

C (Vol 2)- 370-371, 967 


\section{$\mathbf{R}$}

Radiosonde-

C (Vol 1)- 189, 313

Radiosonde observation (RAOB) -

C (Vol 1)- 189, 236, 313

Rain-on-snow (ROS) flood -

C (Vol 1)- 196, 235

Rain shadow-

$\mathrm{C}(\mathrm{Vol} 2)-554,572,663$

$\mathrm{C}(\mathrm{Vol} 4)-1885$

Ranching (see also: Grazing) -

I- 87

$\mathrm{S}-57$

$\mathrm{C}(\mathrm{Vol} 1)-87$

C (Vol 2) - Appendix 3M

C (Vol 4) - 1769, 1812, 1833, 1892, 1900, 1913, 1933, 1940, 1947, 1951, 1952, 1966, 1973,1987

Range clusters-

I- 105-108, 110, 112 (Figure 39), 117-123, 127, 135

S- 119, 123, 124 (Map 20), 125-126

C (Vol 1)- 20, 275 (Map 2.43), 274-276

C (Vol 4)- 2029

Rangeland health -

I- $14,16,181,184$

$\mathrm{S}-9$

C (Vol 1)- 5, 10, Appendix 1A, 53, 55

C (Vol 2) - 364, 433, 437-441, 521, 526 (Map 3.18), 527 (Map 3.19), 528 (Map 3.20), 534 (Map 3.24), 557 (Map 3.34), 575 (Map 3.38), 576 (Map 3.39), 577 (Map 3.40), 612 (Map 3.41), 613 (Map 3.42), 614 (Map 3.43), 666 (Map 3.44), 767, 799, 801, 805-811, 812 (Map 3.60), 813 (Map 3.61), 886, 889, 893-895, 898,

Rangeland integrity918, 922, 967 (definition)

$\mathrm{H}-11,34$

I- 97, 99 (Figure 34), 116

$\mathrm{S}-116,126$

C (Vol 1)- 43, 62

C (Vol 2) - 413, 415, 417 (Map 3.9), 418, 755, 833-834, 839, 900

Rare and sensitive species (see also: Candidate species; Fish, rare and sensitive;

Threatened or endangered species)-

I- $120,140,147,170-171$

$\mathrm{S}-95-97,134$

C (Vol 1)- 47, 76

C (Vol 2) - 447, Appendix 3M

$\mathrm{C}(\mathrm{Vol} 3)-1075,1110,1142,1160,1176,1185,1201,1260,1267-1268,1275,1282,1285$, $1307,1316,1320,1322,1327,1332,1335,1361,1363-1364,1377$, Appendix 4C, Appendix 4G, 1508, 1519, 1521-1522, 1550, 1649, Appendix 5A

C (Vol 4)- 2044 
Raster-

I- 51, 109-110

$\mathrm{S}-121,125$

$\mathrm{C}($ Vol 1) $-96,137,208$

C (Vol 4) - 2023, 2035-2036, 2042, 2051, 2065-2066

Realized fitness-

$\mathrm{C}(\mathrm{Vol} 3)-1517,1528,1658,1676$

Record of decision (ROD) -

$\mathrm{F}$ - Appendix B

I- Appendix A

$\mathrm{S}-143$

C (Vol 1)- 10, Appendix 1A,

Recovery plan-

I- 34, 171

C (Vol 3)- 1519, 1575, 1577-1578, 1773

Recreation-

F- 26, 31, Appendix B

$\mathrm{H}-7,9,18,24$

I- $11,15,21,31,32,40,43,53-54,59,80$ (Figure 24), 82, 84, 86, 87, 125, 127, 128, 130-131, 138, 140, 148, 154, 156, 157, 163, 168, 169, 172, 180, 183, 187, Appendix A, Appendix B

S- 14, 32-33, 35, 38, 41-44, 52, 54, 57, 58, 94, 97, 105, 129, 133, 134, 138, 140

C (Vol 1) - 19, 20, 48, 69, 75, 77, 81-91, 234

C (Vol 2)- 371, 441, 764, 886, 916, Appendix 3M

C (Vol 3) - 1073, 1074, 1088, 1089, 1095, 1098-1099, 1102, 1109, 1253, 1264, 1347, 1370, 1375, Appendix 4A, Appendix 4C, 1513, 1559, 1566, 1571, 1575, 1578, 1579, $1584,1601,1619,1621,1623,1649$, Appendix 5F

C (Vol 4) - 1723, 1728, 1732, 1735, 1738, 1739, 1741-1743, 1745, 1746, 1764, 1774-1787, 1790, 1803, 1805, 1808, 1809 (Map 6.3), 1811-1833, 1835-1840, Appendix 6B, Appendix 6C, 1877, 1879, 1881, 1887, 1890, 1894, 1895, 1897-1899, 1905, 1906, 1908, 1912-1914, 1918, 1919, 1937, 1940-1942, 1944, 1948, 1949-1950, 1952-1953, 1955, 1957, 1958-1960, 1964, 1966-1969, 1984, 1989, 1990, 2028, 2039, 2040, 2052

Recreation opportunity spectrum (ROS) -

C (Vol 1)- 85

C (Vol 4) - 1778-1781, 1786, 1821, 1839, 1908, 1958-1959, 2028, 2039-2040

Recreation visitor days (RVDs) -

I- 53-54

C (Vol 4)- 1774, 1814

Redband trout (see: Salmonids, key)

Redd-

C (Vol 3) - 1109-1110, 1139, 1184, 1190-1191, 1233, 1279, 1344, 1431

Refugia-

$\mathrm{C}(\mathrm{Vol} 2)-922$

C (Vol 3)- 1113, 1137, 1176, 1191, 1288, 1347-1348, 1357, 1565 
Regeneration-

$\mathrm{I}-61$

$\mathrm{S}-34,76,81-82$

C (Vol 1)- 62, 206

C (Vol 2) - 393, 411, 467, 471, 479-481, 490, 497, 502, 505, 549, 467, 588, 597, 605, 641, $650,658,818-819,823,828-829,849,852,886,891$, Appendix $3 \mathrm{~F}$

C (Vol 3) - 1128, Appendix 4B, 1536, 1611, 1653, Appendix 5F

Reptiles-

C (Vol 4)- 1798, 1889

$\mathrm{H}-21$

I- 58, 76, Appendix C, Appendix D, Appendix E

$\mathrm{S}-94,99$

C (Vol 1)- 74-75, 77, 333

C (Vol 3) - 1507, 1509-1511, 1518, 1522-1523, 1531, 1534, 1556, 1569, 1576, 1582-1583, $1585-1586,1591,1600,1612,1618,1621,1637-1638,1645,1655-1556$

C (Vol 4) - 2033

Research natural areas (RNAs) (see: Natural areas)

Research needs/Information gaps-

$\mathrm{F}$ - 8, Appendix B

I- 170, Appendix A

C (Vol 1)- 11, 70-71

$\mathrm{C}(\mathrm{Vol} 2)-805$

C (Vol 3) - 1075, 1267, 1375-1377, 1508, 1648,1650, 1656

C (Vol 4)- 2046

Resiliency/Resilient (see also: Community resiliency; Economic resiliency; Social resiliency) -

$\mathrm{F}-4,9-11,17-18,20-21,37,46$

$\mathrm{H}-6-8,11-12$

I- $12-13,15,19,29-30,33,35-36,68,86,95-96,100,103,108,115,119,121,124-125$, 126 (Figure 42), 127-128, 129 (Figure 43), 134-135, 137, 148, 153-155, 161, 164, 168, $181,183,185,187$, Appendix B

S- $18,24,42-43,45-46,57,113,115-116,126,129,130$ (Map 21), 131, 133, 138, 140-142

C (Vol 1)- 11, 13-14, 16, 24, 43, 48, 50-51, 56-57, 62, 66, 68, 71, 83-84, 89, 89-90, 108, $149,164-165,245,247-249,251-60,264,267-269,274,276$

C (Vol 2) - 365, 367, 371-373, 386-387, 389, 445, 447, 819, 879-881, 907, 918, 921, 923-926, 963-965, 967, Appendix 3M

C (Vol 3)- 1109, 1112, 1142, 1198, 1212, 1223, 1236, 1239, 1262, 1264, 1341-1342, 1345, $1357,1359-1360,1376$

C (Vol 4) - 1738, 1808, 1810-1811, 1833, 1837-1838, 1894, 1930, 1933-1934, 1938-1939, 1942-1950, 1952-1953, 1955-1956, 1975 
Resolution-

$\mathrm{F}-3,13-15,26,46$

I- $24,27,41-43,75,100,113,170,173,180$

S- $100,127,138$

C (Vol 1)- 8, 13-14, 24, 77-78, 97, 130, 172, 188-189

C (Vol 2) - 374, 395, 405, 407-411, 413-414, 460, 470, 481, 511-512, 673, 696, 699, $733-734,761,820,880,886,890-892,895-897,919$

C (Vol 3) - 1085, 1141, 1149, 1173, 1183, 1194, 1214, 1221, 1253-1254, 1262, 1360, 1364, $1379-1380,1524,1527,1530,1537,1618,1628,1639,1643,1645,1647,1655$, $1657-1658$

C (Vol 4)— 2023-2029, 2033-2037, 2048, 2054, 2056-2057, 2066

Retrogression-

C (Vol 2) - 387, 402, 422-423, 425, 428-429, 461, 463-464, 468-469, 471-475, 477, $513-516,553,567,590,600,605,610,615,624,628,635,638,643,650-651$, $655,667,670-671,765,818,820-824,883,908,967$, Appendix 3M

Revegetation-

$\mathrm{C}(\mathrm{Vol} 2)-504,778-779,800-801$

$\mathrm{C}(\mathrm{Vol} 3)-1084$

Ridge (meteorologic) -

Riffle-

$\mathrm{C}(\mathrm{Vol} 1)-188,311-314$

C (Vol 1)- 43, 144, 146-148, 259

C (Vol 3) - 1118, 1123, 1134-1135, 1211, 1271, 1288-1289, 1307, 1325, 1327, 1330, 1332

Riparian habitat conservation areas (RHCAs) -

$\mathrm{S}-104$

C (Vol 1)- 70

C (Vol 3) - 1354, 1364-1369, 1371, 1374

Riparian herbland -

$\mathrm{I}-106$

C (Vol 2) - 414, 507-508, Appendix 3B, Appendix 3C, Appendix 3D

Riparian herb potential vegetation group (PVG) (see: Potential vegetation group, riparian herb)

Riparian management objectives (RMOs) -

C (Vol 3)- 1371

Riparian shrub potential vegetation group (PVG) (see: Potential vegetation group, riparian shrub)

Riparian woodland potential vegetation group (PVG) (see: Potential vegetation group, riparian woodland)-

Risk-

analysis-

$\mathrm{F}-20,33$

C (Vol 2)- 390, 392, 916

$\mathrm{C}(\mathrm{Vol} 3)-1515$

assessments-

F- 9, 23, 26, 32-35, Appendix B

I- 39

management-

$\mathrm{F}-20,23,32-35$

I- 15-16, 39-41, 157, 184

C (Vol 1)- 16

$\mathrm{C}(\mathrm{Vol} 2)-392$ 
Road(s) (see: Road density; Roadless areas)

Road density-

$$
\mathrm{H}-19
$$

I- 15, 62, 66 (Figure 15), 67 (Figure 16), 68, 72 (Figure 20), 73, 94, 97, 108-110, 113-115, $117,119,121,140,146-147,149,183$, Appendix B

S- 52, 54, 84 (Map 9), 85, 87, 104-106, 110 (Map 17), 121-123, 125-128, 134, 139-140

C (Vol 1)- 60, 62, 64-66, 69, 85, 90, 152, 214, 230, Appendix 2A

C (Vol 2) - 404, 415, 418-419, 530-531, 541 (Map 3.28), 542 (Map 3.29), 556, 573, 585, $593,603,611,621,626,636,646,656,664,834,882,900,914-915$

C (Vol 3) - 1104, 1125, 1130, 1133-1137, 1139, 1172-1173, 1177, 1183, 1194, 1200, 1239, $1253-1256,1258-1259,1262,1345,1347,1372-1373,1379,1620,1622$

C (Vol 4)- 1778, 1786, 1828-1829, 1840, 1962, 2025, 2029, 2031, 2038-2040, 2054-2056

Roadless areas-

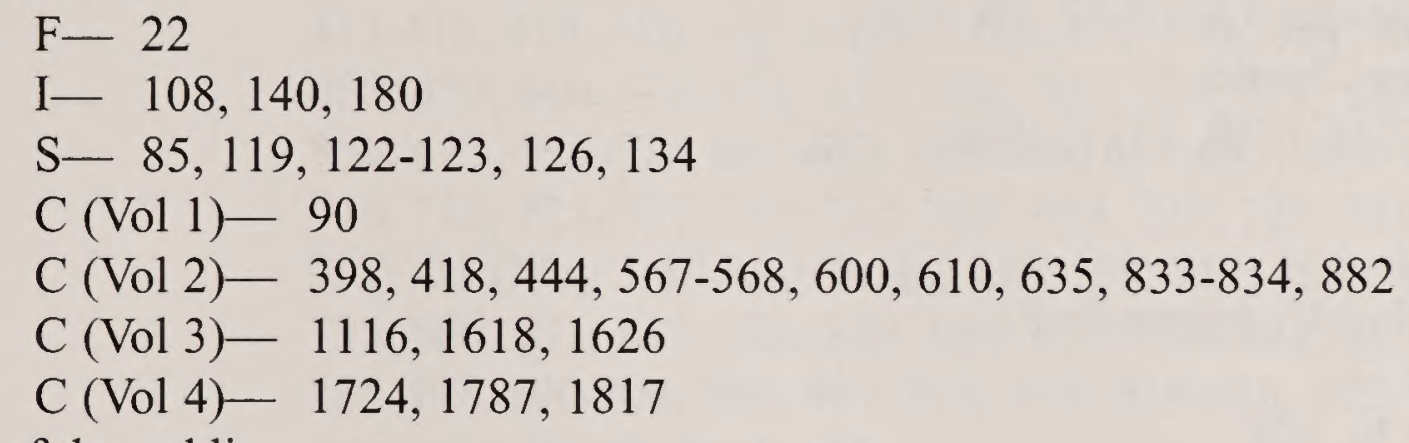

Role of the public-

$\mathrm{S}-26-28,59$

C ( Vol 1)- 87, 89-92

Rotation-

C (Vol 4)- 1881, 1919, 1971-1977

$$
\begin{aligned}
& \text { C (Vol 1)- Appendix 2A } \\
& \text { C (Vol 2)- } 767,772-774,804 \\
& \text { C (Vol 3)- } 1566,1653 \\
& \text { C (Vol 4) } 1798
\end{aligned}
$$

Runoff-

$$
\begin{array}{ll}
\text { S- }-86 & \\
\mathrm{C}(\text { Vol 1)- } & 147,163-164,194,230 \\
\mathrm{C}(\text { Vol 2)- } & 366,403,777-779,781,785,819,821 \\
\mathrm{C}(\text { Vol 3)- } & 1084,1088,1098-1101,1650
\end{array}
$$

Rural/wildland interface (see: Wildland interaction/Wildland interface)

\section{$\mathbf{S}$}

Sagebrush grassland (see: Altered sagebrush steppe)

Salinity-

C (Vol 2) - 437-439, 525, 557 (Map 3.34), 577 (Map 3.40), 610, 612-614 (Map 3.41-Map 3.43), 620, 663, 666 (Map 3.44), 807-808, 811, 812 (Map 3.60)

Salmon/Salmonids (see: Salmonids, key) 
Salmonids, key-

bull trout-

F- Appendix B

$\mathrm{H}-16$

I- $68,73,123,147$, Appendix A, Appendix E

S- 104, 106-107, 112

C (Vol 1)- 10, Appendix 1A, 67, 70, 137, 328

$\mathrm{C}$ (Vol 3)- Aquatics-Chapter 4

$\mathrm{C}(\mathrm{Vol} 4)-2026$

chinook salmon-

$\mathrm{H}-16$

I- $68,73-74,116-117,123,147$, Appendix E

S- 46, 106-107, 112

C (Vol 1) - 10, Appendix 1A, 65-67, 70, 328

$\mathrm{C}$ (Vol 3) - Aquatics-Chapter 4

C (Vol 4) - 1746, 1748-1749, 1819, 1889

redband trout-

$\mathrm{H}-16$

I- $68,73,117,123,147$, Appendix E

S- 104, 106-107, 112

C (Vol 1)- 65, 67, 70, 328

C (Vol 3) - Aquatics-Chapter 4

steelhead-

$\mathrm{H}-16$

I- $34,68,73,117,123,147$, Appendix E

S- 29, 46, 104, 106-107, 111-112

C (Vol 1)- 65, 67, 328

C (Vol 3) - Aquatics-Chapter 4

C (Vol 4) - 1746-1749, 1780, 1818, 1883, 1889, 1914

westslope cutthroat trout-

$\mathrm{H}-16$

I- $68,73,147$, Appendix E

$\mathrm{S}-104,106,112$

$\mathrm{C}(\mathrm{Vol} 1)-65,67,328$

$\mathrm{C}$ (Vol 3) - Aquatics-Chapter 4

yellowstone cutthroat trout-

$\mathrm{H}-16$

I- $68,73,147$, Appendix E

S- 104, 106, 112

$\mathrm{C}($ Vol 1) - 65-67, 328

$\mathrm{C}(\mathrm{Vol} 3)$ - Aquatics-Chapter 4

Salvage-

I- 16, 178-180, 184

$\mathrm{S}-22$

$\mathrm{C}(\mathrm{Vol} 2)-924$

$\mathrm{C}(\mathrm{Vol} 3)-1654$

C (Vol 4) - 1789, 1796-1798, 1827-1828, 1891, 1910-1912, 1981 
Saprophyte-

C (Vol 3)- 1535, 1676

Scale, general-

$\mathrm{F}-13-15$

I- $41-43$

$\mathrm{C}(\mathrm{Vol} 1)-14$

$\mathrm{C}(\mathrm{Vol} 2)-373-374$

broad-scale (broadscale)-

I- $75,96,105,106,142$

$\mathrm{S}-93,99,116,135$

C (Vol 1) - 14, 51-62, 63, 69, 73, 77, 97-98, 127, 129, 130, 133, 135, 151, 152, 285, Appendix 2A

C (Vol 2) - 374, 383-385, 388, 389, 395, 396, 398, 399, 400, 402, 404, 407, 410, 411, 413-415, 418, 420, 430 (Map 3.10), 431, 433, 434, 438, 439, 441, 442, 449, $458,459,460,470,476,477,496,508,509,511,536,538,541$ (Map 3.28), $560,673,675-678,682-683,686-689,692-697,699-700,703,705,706,708$, $710,712,715,717,719,721,724,726,727,729,731,732-734,735-736$, 737-754 (Map 3.47-Map 3.58), 756, 758, 759, 760, 761, 766, 785, 793, 794, $795,805,811,815,820,829,845,875,880,881,883,885,886,890-892$, 895-897, 900-904, 906, 907, 914, 916, 919-920, 922, 929, Appendix 3B, Appendix 3C, Appendix 3E

C (Vol 3) - 1073, 1074, 1086, 1130, 1135, 1139, 1141, 1146, 1150, 1174, 1253, 1262 , 1347, 1356, 1360, 1370, 1376, 1379, Appendix 4A, Appendix 4C, 1523, 1524, $1531,1567,1573,1582,1585,1586,1588,1589,1627,1628,1639,1644$, $1646,1657,1658$

C (Vol 4)- 1878, 1898, 1957, 1964, 2024-2026, 2031, 2033, 2037, 2039, 2042, 2043, 2054, 2056, 2057, 2058, 2059

fine-scale-

$\mathrm{H}-20$

I- 145

$\mathrm{C}($ Vol 1) - 51-52

C (Vol 2) - 373-374, 383, 395-396, 398, 402-404, 420, 432, 466, 511, 532 (Map 3.22), 670, $778,794,824,880,907,919-920,922,962$

C (Vol 3)- 1339, 1364

C (Vol 4)- 2045, 2054, 2056

mesoscale-

C (Vol 1)- 200, 312

mid-scale-

$\mathrm{H}-20$

C (Vol 1)- 14, 51-54, 64, 66, 151-152, 157

C (Vol 2) - 374, 383, 395-396, 398-400, 404, 410, 418, 420, 431-432, 508, 560, 573, 696-697, 733-734, 820, 876, 880, 886-887, 902-906, 914, 919-920

C (Vol 3) - 1085-1087, 1267, 1339, 1350, 1360, 1370, 1524-1527, 1531, 1585, 1590, 1628,1646

C (Vol 4)- 1908, 2021-2022, 2029, 2033, 2054, 2056-2058 


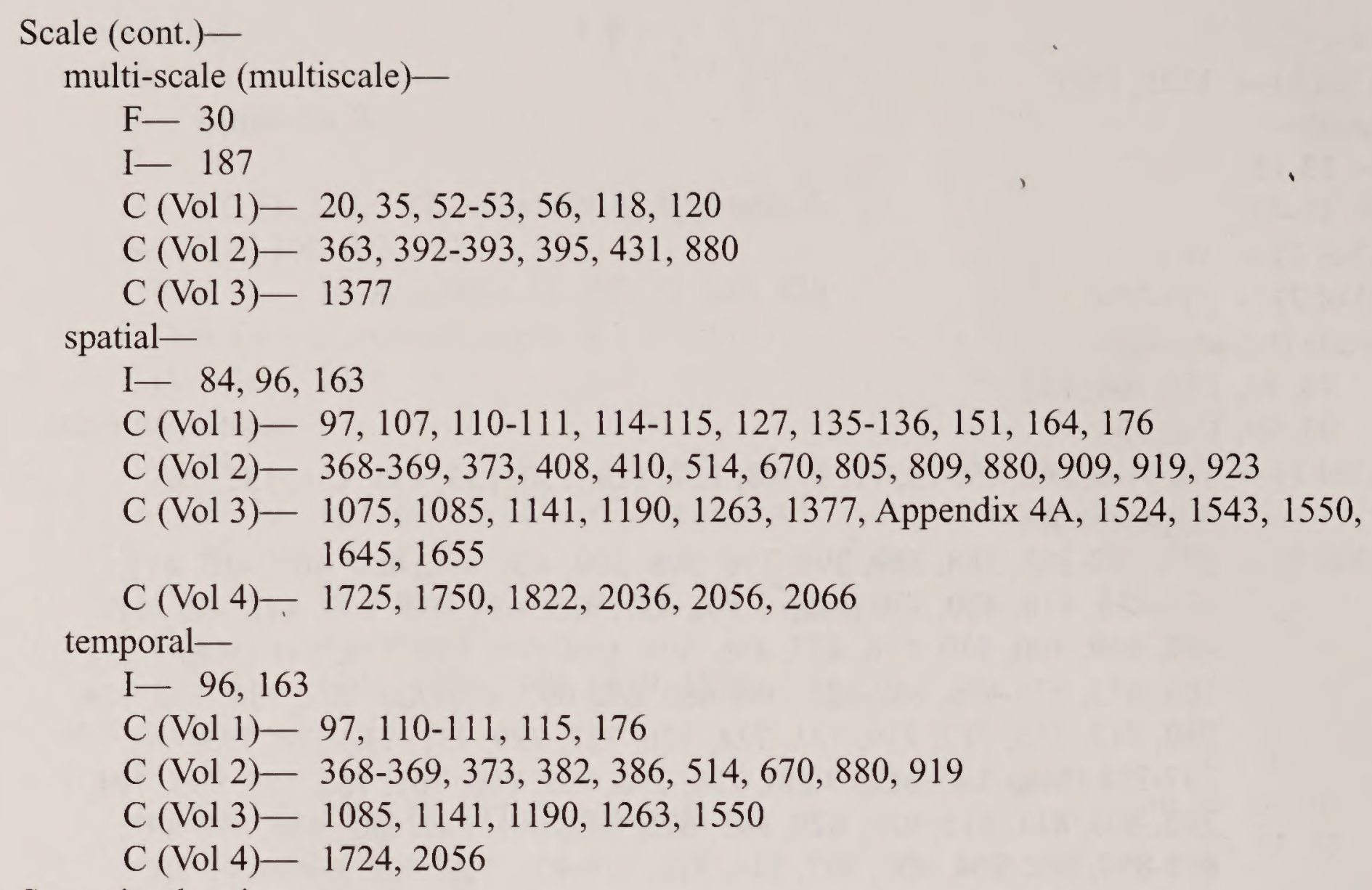

Scenario planning-

$\mathrm{F}-5,26,33,46$

C (Vol 1)- 24

Scenery/Scenic integrity-

$\mathrm{F}-21-22$

$\mathrm{H}-28$

I- 37-38, 91, 93 (Figure 32), 94, 131, 145-146, 157, 168

$\mathrm{S}-33,52,54,58,139-140$

$\mathrm{C}(\mathrm{Vol} 1)-86,90-91$

C (Vol 4) - 1724, 1814, 1817-1818, 1829-1830, 1840, 1881, 1887, 1905, 1908, 1944, 1949, 1957, 1960-1962, 1964, 1965 (Map 7.4), 1966, 1968, 2025, 2039-2040

Science Integration Team (SIT) -

$\mathrm{F}$ - Appendix C

$\mathrm{H}-\mathrm{vi}, 4$

I- iv, v-viii, $11,17,19,22,24,26,30,47,84,95,137,149,163,175,177,179,181$, Appendix D

S- 94

C (Vol 1)- 8, 11, 13-14, 16, 74, 95, 108, 114, 116, 171-172, 175, 207

C (Vol 3) - 1432, 1508, 1516, 1519, 1521, 1533-1534, 1548, 1579

C (Vol 4) - 1723, 1827, 1832, 2017-2018, 2020-2022, 2032, 2034, 2036, 2040, 2050, 2059

Scientific assessment-

F- Appendix B

$\mathrm{H}-4,12,27,34$

I- 17, 22, Appendix A

C (Vol 1)- 8, 11, 13-14, 16, 19, 95

$\mathrm{C}(\mathrm{Vol} 3)-1073$

$\mathrm{C}(\mathrm{Vol} 4)-2046$ 
Section (ecological) -

C (Vol 1) - 16, 113, 122, 128-130, 133, 135, 175, 201-203, 249, 252, 256, 258, 313-314

$\mathrm{C}(\mathrm{Vol} 2)-374,376$ (Map 3.1), 410, 517, 585, 592, 601, 610, 620, 635, 643, 655, 965

$\mathrm{C}(\mathrm{Vol} 3)$ - Appendix 4A

Sedimentation-

$\mathrm{I}-30,100,164$

S- $105,107,116$

C (Vol 1)- 68-69, 108, 112, 114, 151, 157, 176, 179

C (Vol 2)- 391, 512, 965, Appendix 3M

C (Vol 3) - 1087-1088, 1100-1104, 1110, 1139, 1234, 1271, 1289, 1316, 1318, 1325, 1330, $1339,1345-1347,1365,1370,1379,1582,1614$

C ( Vol 4)- 1803

Selective cutting/Selective harvest-

C (Vol 2) - 567, 580, 605, 638, 650, 818, 884

$\mathrm{C}(\mathrm{Vol} 3)-1559$

C (Vol 4)- 1912

Sense of place-

$\mathrm{F}-18,21-23$

$\mathrm{I}-30,36,86,96$

S- $24,38,62,129$

C (Vol 1)- 89

C (Vol 4) - 1907-1908, 1920, 1928, 1936, 1962, 2025, 2031

Sensitive species (see: Endangered species; Fish, rare and sensitive; Rare and sensitive species;

Threatened and endangered species)

Seral stage, general-

$\mathrm{F}-46$

$\mathrm{S}-83$

C ( Vol 1) - 24

$\mathrm{C}(\mathrm{Vol} 2)-402,830-833,966-968$

early-

C (Vol 2)- 488, 493, 650, 828, 830-833, 967 (definition)

$\mathrm{C}(\mathrm{Vol} 3)-1573$

mid-

C (Vol 2)- 481, 488, 490, 830-833, 967 (definition)

late-

C (Vol 2) - 515, 830-833, 967 (definition)

Short-term-

$\mathrm{F}-14-15,19,22,28$

I- $33,41-42,138,140,163-165,170,172,177$

$\mathrm{S}-74,134$

C (Vol 1)- 85, 180, 194, 202-203

C (Vol 2) - 386, 393, 406, 445, 448, 828, 911, 915-918, Appendix 3M

C (Vol 3) - 1239, 1260, 1264, 1339, 1341, 1345, 1356, 1379, 1567, 1654, Appendix 5G

C (Vol 4) - 1758-1760, 1763-1765, 1839

Shrink-swell potential (SSP) -

C (Vol 2) - 437-439, 521-525, 527 (Map 3.19), 528 (Map 3.20), 614 (Map 3.43), 807, 812 (Map 3.60), 813 (Map 3.61) 
Site-

$\mathrm{F}-15,19,21,26,33-36$

$\mathrm{H}-12$

I- $21,40-41,43,45,50,130,169,173-174$

S- 67

C (Vol 1) - 48, 56, 89, 113, 127, 129, 148, 152, 165, 180, 185, 202, 205-206, 229-230, 245

C (Vol 2)- 366-367, 373, 383, 387-388, 399, 401, 406, 443, 449, 465-468, 470-471, 480, $484,492,497,501,504,766,769,777-783,794,798-799,801,806,821-822$, 879,962

C (Vol 3) - 1100-1101, 1105, 1183, 1279, 1365, 1367, 1370, 1535-1536, 1555, 1562, 1566, $1577,1614,1656$, Appendix 5G

C (Vol 4) - 1787, 1796, 1798, 1907-1908, 1969, 2022-2023, 2052

Slope winds-

Smolt-

$\mathrm{C}(\mathrm{Vol} 1)-234,313$

C (Vol 3) - 1108, 1220-1223, 1232, 1234, 1236, 1288, 1343-1344, Appendix 4D

Snag(s) -

I- $14,97,181$

S- $83,85,94,116$

C (Vol 1)- 51-52, 60, 75

C (Vol 2)- 393, 420, 487, 490, 492-493, 824-833, 882, 920, 925, 962, Appendix 3M

C (Vol 3) - 1520, 1536, 1557, 1563, 1571, 1583, 1586, 1600-1606, 1612, 1614, 1647, 1654, 1656, 1658, Appendix 5F, Appendix 5G

Snow water equivalent (SWE) -

Snowfall-

C (Vol 1)- 188, 235-236, 313

C (Vol 1)- 36-37, 194, 199, 234-235, 237-243, 313

Social/economic clusters (socioeconomic clusters)

I- $127-128$

Social resiliency/Social-economic (socioeconomic) resiliency (see also: Community resiliency;

Economic resiliency; Resiliency)-

$\mathrm{F}-17-18,20-21$

$\mathrm{H}-6-7,12$

I- $12-13,15,30,35-36,95-96,124-128,129$ (Figure 43), 134-135, 137, 148, 153-154, $164,181,183,185,187$, Appendix B

S- 24, 129, 130 (Map 21), 131, 133, 138, 140, 142

$\mathrm{C}($ Vol 1)- 16

Sodicity-

$\mathrm{C}(\mathrm{Vol} 2)$ - Appendix 3M

\section{Soil-}

C (Vol 2)- 437-439, 666 (Map 3.44), 807, 812 (Map 3.60), 967

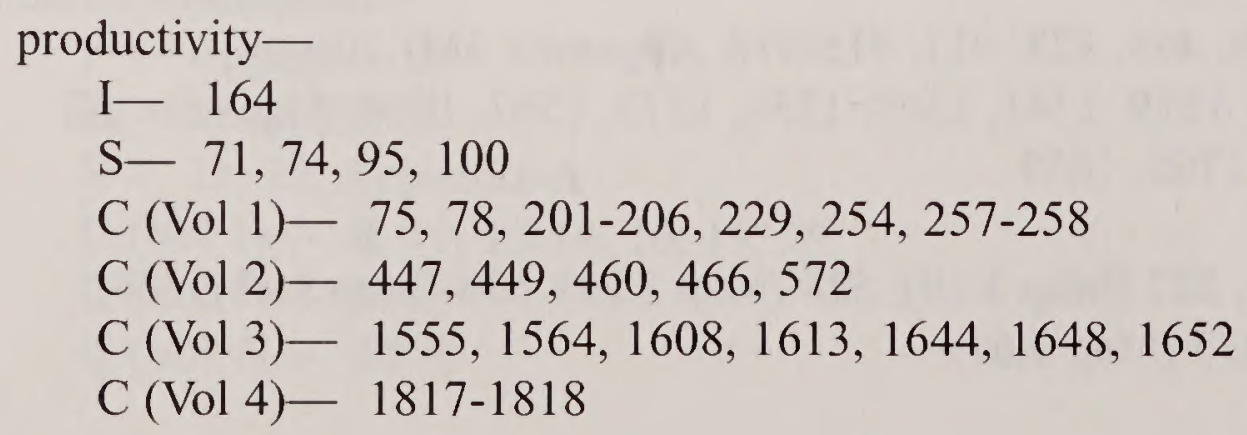


Soil (cont,)-

structure-

I- 100,167

$\mathrm{S}-97$

C (Vol 1)- 76-77, 229

C (Vol 2) - 366, 438, 466

C (Vol 3) - 1100, 1563-1564, 1613, Appendix 5G

Southern Oscillation (SO) -

C (Vol 1)- 310, 314

Spatial unified data dictionary (SPUDD)-

C ( Vol 1) - 96-97

C (Vol 4)— 2031, 2047-2048, 2050, 2066

Species richness-

I- 34

$\mathrm{S}-111$

C (Vol 1)- 66, 116

C (Vol 2)- 776, 798-802, 804

C (Vol 3) - 1146, 1150-1151, 1239, 1241, 1249, 1262, Appendix 4C, 1531, 1615, 1646,1655

Stable air-

C (Vol 1)- 314

Stakeholders-

F- 4-5, 8-9, 15, 19, 25-27, 29-31, 33, 36, 46

I- $12,20,22,38,45,161,164,173$

$\mathrm{S}-23,25-26$

C (Vol 1)- 25, 91-92

C (Vol 4) - 1830, 1903, 1919, 1972, 1982, 1985, 1988

Steelhead (see: Salmonids, key)

Stronghold-

$\mathrm{H}-19$

I- 15-16, 68, 71 (Figure 19), 72 (Figure 20), 73-74, 103, 108, 113-117, 119-120, 123, 146, 179-180, 183-185

S- 110 (Map 17), 111-112, 127-128, 142

C (Vol 1) - 52, 67, 70

C (Vol 2) - Appendix 3M

C (Vol 3) - 1172, 1185, 1200, 1239, 1249, 1263, 1339, 1343, 1347-1351, 1354, 1356-1357, 1358 (Map 4.74), 1359, 1361, 1374, 1472

Structural stage-

I- 105,166

$\mathrm{S}-80-81$

C (Vol 2)- 401, 403, 405-406, 410, 441-442, 470, 488, 492, 598, 824-825, 883, 906, 962, 967, Appendix 3B, Appendix 3C, Appendix 3D, Appendix 3F, Appendix 3G

C (Vol 3) - 1126, 1128, Appendix 4B, 1524, 1658

C (Vol 4) - 2039-2043

Subbasin (see: Hydrologic hierarchy; Hydrologic unit codes; Scale) 
Subregional/Subregions-

$\mathrm{F}-14-15$

I- 62,83 (Figure 25), 96

$\mathrm{S}-42,129$

C (Vol 1) - 16, 35, 83, 113-114, 118, 120, 122, 127, 138, 140, 141 (Map 2.8) 3 142-143, 145, $149,163-134,172,175,201,207,214,269,313-314$

C (Vol 2) - 366-367, 371, 373, 391-393, 670, 785, 905, 965, Appendix 3M

C (Vol 4)- 1725, 1814, 1837, 1961

Subsection (ecological) -

$\mathrm{I}-60$

C (Vol 1) - 16, 35-42, 113, 116, 118, 119 (Map 2.3), 120, 122-128, 130, 140, 142-143, 149, $151-152,175,208,212,214,231,245-248,250-251,253-258,314$

C (Vol 2) - 404, 420, 430 (Map 3.10), 431, 856

C (Vol 4)- 1908, 1961-1962, 2027, 2029, 2038, 2054

Subwatershed (see: Hydrologic hierarchy; Hydrologic unit codes; Scale)

Successional path -

C (Vol 2)- 967-968

Sustainability -

F- 18, Appendix B

I- $13-14,17,54,75,155,185$, Appendix A

$\mathrm{S}-140$

C (Vol 1)- 8, 10, Appendix 1A, 56

C (Vol 2) - 363-364, 385, 390, 393, 804, 908, 925

C (Vol 3)- 1508, 1528, 1643, 1647, 1652

C (Vol 4)- 1724, 1805, 1816, 1832-1833, 1952, 1986, 2052

Symbiont-

C (Vol 3)- 1432, 1535, 1676

Sympatric-

$\mathrm{C}(\mathrm{Vol} 2)-370$

C (Vol 3) - 1149, 1192, 1200-1201, 1202 (Map 4.16), 1204-1207, 1208 (Map 4.18), 1210$1211,1213,1246,1248,1261,1432$, Appendix 4D

Synoptic-

C (Vol 1)- 144, 188, 314

Synoptic weather

C (Vol 1)- 188, 314

Systems modeling-

$\mathrm{F}-30,46$

C (Vol 1)- 25 


\section{$\mathbf{T}$}

Taxon-

$\mathrm{I}-75$

C (Vol 1)- 73

C (Vol 3) - 1291, 1298, 1432, 1521, 1529, 1533, 1541, 1550, 1591, Appendix 5D

Tectonic-

$\mathrm{C}($ Vol 1) -179

C (Vol 3)- 1081

Terrestrial community type/Terrestrial community group (see: Vegetation classifications)

Terrestrial ecology-

I- $47,74,144-145,182-183$

S- 93, 100, 136

C (Vol 1)- 20, 75

C (Vol 3) - Terrestrial-Chapter 5, 1339, 1508-1509, 1544, 1585, Appendix 5A

Terrestrial habitat-

$\mathrm{H}-29$

I- 149,185

$\mathrm{S}-116,142$

C (Vol 2)- 399, 968

Terrestrial integrity-

I- 123

C (Vol 2)- 968

Theme/Theme group (geographic) -

I- 115,117

C (Vol 1)- 97, 151, 175

$\mathrm{C}(\mathrm{Vol} 2)-436$

$\mathrm{C}(\mathrm{Vol} 3)-1126$

C (Vol 4) - 2023-2029, 2031-2033, 2048-2051, 2055, 2057, 2065-2066

Thermal cover-

$\mathrm{C}(\mathrm{Vol} 2)-776$

C (Vol 3)- 1100

Thermal low (low heat)

C (Vol 1)- 235, 311, 314

Thinning-

I- 149,169

$\mathrm{S}-76,140$

C (Vol 1)- 55

C (Vol 2)— 443, 447, 476-477, 487, 777, 819, 828, 847-854, 867, 915-917, 921, 966, Appendix $3 \mathrm{~L}$

C (Vol 3) - 1340, 1539, 1562, 1575, 1654

C (Vol 4) - 1798, 1894, 1911, 1957 
Threatened or endangered species (TES) (see also: Candidate species; Endangered species/Endangered Species Act; Fish, rare and sensitive; Rare and sensitive species) -

F- 19, 24, Appendix B

$\mathrm{H}-21,32$

I- $14,16,57-59,75,103,140,166,171,182,184$, Appendix A

S- 93-94, 97, 106, 134

C (Vol 1) - 10, Appendix 1A, 47, 65, 73-77

$\mathrm{C}(\mathrm{Vol} 2)-772,908,922-923$, Appendix 3M

C (Vol 3) - 1073-1074, 1081, 1106, 1141, 1146, 1148, 1160-1161, 1193, 1222-1223, 1237, $1267,1270,1285,1289,1291-1292,1307,1311,1314,1332,1337,1347-1348$, $1359,1507-1508,1511,1519,1523,1533-1535,1543-1544,1550,1568$, $1575-1576,1578,1581,1628,1643-1644$

C (Vol 4) - 1773, 1818, 1905, 1913, 1969, 2044, 2051

Timber (see also: Logging) -

F- 19, 22, 26, 27, Appendix B

$\mathrm{H}-12-14,17,22,23,28,31,33$

I- $15,16,32,35,37,44,54-55,61,63,81,82,84,85$ (Figure 26), 86, 89 (Figure 29), 108, $116,119,125,138,140,142,148,155,161,163,164,168,172,178,183,185$, Appendix A

S- $22,31,32,37,40,41,46,52,55-57,75-77,80,82-83,96,133-134,138-141$

C (Vol 1) - 20, Appendix 1A, 54, 57, 60, 62, 64, 69, 76, 81-82, 84-86, 87, 89, 193, 204, 206, 229

C (Vol 2) - 393, 418, 441, 443, 447, 460, 462, 466, 468, 473, 488, 493, 496, 514-515, 530, $531,553,560,563,581,585,586,590,593,597-598,602-603,605,608,615$, $628,635,636,638,641,643,646,650-651,655-656,658,661,670-671,696$, $733,753,755,762,817,818,828,829-833,880-882,884-885,892,896,898$, 900, 901, 903-906, 915-916, 921, 924, Appendix 3M

C (Vol 3) - 1085, 1088-1089, 1100-1102, 1116, 1118, 1139, 1161, 1174, 1175, 1188, 1192 , $1212,1222,1234,1253,1264,1307,1325,1327,1335,1337,1340,1342$, $1346,1347,1361,1370,1372,1373$, Appendix 4A, Appendix 4C, 1511, 1543, $1570,1575,1578,1579,1590,1611,1648,1649,1653,1656$

C (Vol 4) - 1723-1724, 1728, 1729, 1732, 1737, 1739, 1744, 1745, 1789-1799, 1805-1833, 1835-1840, Appendix 6C, 1879, 1885, 1888-1889, 1890-1892, 1905-1906, $1910,1917,1919,1925,1930,1936-1937,1938,1941-1942,1949-1954,1955-$ $1956,1957,1961,1989,1990,2025,2046$

Topography-

$\mathrm{H}-3$

I- 18 (Figure 1), 48, 120

S- 12 (Map 1), 67, 75

C (Vol 1) - 56, 118, 122, 176, 180, 185, 188-189, 194, 198, 200-201, 234, 313

C (Vol 2) - 366, 375, 382, 386, 388, 531, 554, 556, 560, 568, 573, 581, 585, 592, 602, 610, $615,620-621,624,626,635,643,646,655,661,664,670,753,815,818,824$. $868,880,966$

C (Vol 3)- 1075-1077, 1084, 1100, 1137, 1359, 1371, 1542

C (Vol 4)- 2035

Transpiration-

C (Vol 1) - 229-230, 310

C (Vol 2) - 366, 368, 383, 389, 405, 405, 783, 819, 823, Appendix 3M

$\mathrm{C}(\mathrm{Vol} 3)-1562$ 
Tribal/Tribes (see also: American Indians) -

F- 3-5, 27, 29, 31, 46, Appendix B

$\mathrm{H}-25$

I- $11-12,17,21-22,26,29,47-48,50-53,75,91,138,140,145,167-168,171,177-178$, 185, Appendix A

S- 22-23, 26, 29-30, 61-66, 93-94, 134, 138-139, 142

C (Vol 1)- 7-8, 25, 73-74, 76, 78, 87, 91, 95

C (Vol 2)- 396, 597, 965

C (Vol 3) - 1086, 1109, 1115, 1118, 1130, 1132, 1147, 1213, 1220, 1222, 1235, 1253, $1268,1278,1282,1284,1311,1354$, Appendix 4D, Appendix 4F, 1507-1508, $1521,1531,1533-1534,1543,1548,1550,1574,1579,1581,1644$

C (Vol 4) - 1723, 1729-1730, 1746-1747, 1749-1755, 1787-1788, 1799, 1879-1880, $1883-1884,1890,1894,1915,1921,1923-1932,1973,1979,1985,1987-1988$, $2018,2028,2038-2040,2054$

Trough (meteorologic) -

$\mathrm{C}($ Vol 1)- 39, 188, 234, 311-312, 314

True firs -

C (Vol 4)- 1796

Turbidity-

$\mathrm{S}-104$

C (Vol 1)- 63

C (Vol 3)- 1093, 1222, 1234

$\mathbf{U}$

Underburn (see also: Fire, ground/surface)-

I- 172

$\mathrm{S}-81$

C (Vol 2)- 443, 481, 484, 488, 490, 492-493, 580, 615, 891, 896

Understory-

I- $117,119,165,169-170,172$

$\mathrm{S}-79-83,123$

C (Vol 2)- 387, 403, 412, 467, 481, 500, 502, 505, 514, 516, 563, 580, 621, 624, 628-629, $650,664,697,766,775-783,793,816,821-823,828,883,887,890-892,905$, Appendix 3B, Appendix 3C, Appendix 3D, Appendix 3F, Appendix 3G

C (Vol 3) - 1126, 1128, Appendix 4B, 1526, 1556, 1563-1564, 1568, 1570, 1601, 1650

Uneven-aged management-

$\mathrm{C}(\mathrm{Vol} 3)-1536$

Ungulates-

$\mathrm{F}-18$

I- 114, 117, Appendix C, Appendix D

S- $49,83,128$

C (Vol 2)- 497, 499, 501, 507, 509, 511, 629, 767, Appendix 3M

C (Vol 3) - 1513, 1522-1523, 1571-1575, 1577-1578, 1580-1581, 1584, 1609, 1646, 1656, Appendix 5G

C ( Vol 4)- 1887

Unstable air-

$\mathrm{C}($ Vol 1) $-235,314$ 
Upper air-

C (Vol 1)- 199, 314

Urban/wildland interface (see: Wildland interaction/Wildland interface)

V

Vascular plants-

$\mathrm{H}-21$

I- 58, 144, Appendix C

$\mathrm{S}-94,96$

$\mathrm{C}(\mathrm{Vol} 1)-74,76,78$

$\mathrm{C}(\mathrm{Vol} 2)-801-802,804$

C (Vol 3) - 1507-1508, 1510-1511, 1519, 1521, 1529, 1531, 1533-1534, 1538-1542, 1550, 1610, 1614, 1618, 1644, 1651-1652, Appendix 5A, Appendix 5G

Vector/Vector data (geographic) -

C (Vol 1)- 96, 149, 151

C (Vol 4) - 2023, 2035-2036, 2051, 2054, 2066

Vegetation classifications-

general-

$\mathrm{C}(\mathrm{Vol} 2)-962$

$\mathrm{C}(\mathrm{Vol} 3)-1086,1151,1524-1525$

C (Vol 4) - 2054

physiognomic type/physiognomic type group-

$\mathrm{C}(\mathrm{Vol} 1)-54,128$

C (Vol 2) - 398-400, 432, 449, 480-480, 485-487, 489-495, 499-500, 502, 503, 505-510, $531,548-555,560,562-571,579,581-591,593,595-602,605,609,615$, $617-619,621,623-625,628-635,639-654,658-662,664,667,670,734,829$, 834, 842-846, 849, 852, 882, 884-885, 905, 966 (definition), Appendix 3C, Appendix 3D, Appendix 3H, Appendix 3K, Appendix 3L, Appendix 3N

potential vegetation classifications-

C (Vol 1)- 127, 128-130, 171, 259

potential vegetation group (PVG) (see: Potential vegetation group)

potential vegetation type (PVT)-

$\mathrm{S}-385$

C (Vol 1)- 35, 38, 104, 213, 231, 248

C (Vol 2) - 374, 383, 398-403, 408-410, 432, 441-442, 456-457, 459-460, 468, 469, 764-766, 781, 795, 803 (Map 3.59), 805, 846, 849, 852, 906, 966, Appendix 3A, Appendix 3F, Appendix 3M

C (Vol 3) - 1126, 1133-1134, Appendix 4B

C (Vol 4) - 2039, 2040

terrestrial community type/tperrestrial community group-

I- 105,108

S- 63, 166, 136

C (Vol 1) - 53-54, 62

C (Vol 2) - 409-411, 413-415, 432, 449, 699-701, 703-705, 707-734, 735-736, 737

(Map 3.47), 738 (Map 3.48), 739, 741, 742-749 (Map 3.49-Map 3.57), 753, 754 (Map 3.58), 759, 760, 761, 880, 889, 893, 897-899, 901, 968, Appendix 3B

C (Vol 4) - 1930 
Vegetation composition-

$$
\begin{aligned}
& \mathrm{F}-13 \\
& \mathrm{H}-31 \\
& \text { I- } 43 \\
& \text { S- } 75 \\
& \text { C (Vol 1)- 57, 60, } 205 \\
& \text { C (Vol 2) - 374, 401-402, 420, 447, 462, 466, 481, 488, 492, 496, 556, 624, 764, 769, 815, } \\
& 855,880-881,885-886,901,906,922 \\
& \mathrm{C}(\mathrm{Vol} 3)-1575
\end{aligned}
$$

Vertebrate(s) -

$\mathrm{F}-16,22$

I- $29,34,51,57,75,144$, Appendix D

S- 94-95, 100

C (Vol 1) - 75, 78

C (Vol 2) - Appendix 3J

C (Vol 3) - 1509, 1511, 1513, 1522-1524, 1526-1527, 1529-1532, 1539, 1559, 1561, 1563, $1565,1568,1572,1574-1576,1578,1585-1587,1589,1591,1598$ (Map 5.2a), 1599 (Map 5.2b), 1601, 1611-1612, 1616 (Map 5.3a), 1617 (Map 5.3b), 1626, $1628,1637-1639,1641,1644-1646,1654$, Appendix 5G

C (Vol 4) - 2044

Viable/Viable population-

$\mathrm{F}-4,10,16,18-20,22-23,46$

$\mathrm{H}-25$

I- $12,29-30,33-34,96,165-166,170-171,173$

$\mathrm{S}-22,24$

$\mathrm{C}(\mathrm{Vol} 1)-12,24-25,87$

C (Vol 2)- 442, 908, 921, 925, 963

C (Vol 3) - 1344, 1507, 1513, 1535, 1572, 1627, 1676

Virtual system-

$$
\begin{aligned}
& \mathrm{F}-46 \\
& \mathrm{C}(\mathrm{Vol} 1)-25
\end{aligned}
$$

Volcanos/Volcanics/Volcanism-

$\mathrm{F}-4,10,12$

I- 27, 32-33, 48

$\mathrm{S}-18,21,71,74-75$

C (Vol 1)- 37-40, 42, 114-116, 124-126, 143, 176-180, 200-202, 204, 211, 245-247, $249-254,257-258$

C (Vol 2) - 366-367, 369-370, 372, 382, 390, 517, 568, 585, 602, 610, 620, 661, 671, $873-874,880,968$

C (Vol 3) - 1075-1077, 1081, 1088, 1127, Appendix 4A, 1533, 1619, 1621, 1637, Appendix 5F

C ( Vol 4) - 2027 
W

Water allocation-

C (Vol 1)- 86

C (Vol 4) - 1799-1800, 1802-1803, 1841

Watershed (see: Hydrologic hierarchy; Hydrologic unit codes; Scale)

Water storage-

$\mathrm{C}(\mathrm{Vol} 2)-769,781$

$\mathrm{C}(\mathrm{Vol} 3)-1093,1098,1100$

Weed(s) -

$\mathrm{F}-46$

$\mathrm{H}-1,14-15$

I- $16,114,117,119-121,123,140,143,149,153,164-165,171,184$

S- $22,79,85,87,88$ (Map 10), 128, 140

$\mathrm{C}(\mathrm{Vol} 1)-25$

C (Vol 2)- 403, 433-435, 437, 444, 459, 505, 416, 459, 763, 765, 782-785, 796, 800-801, 914, Appendix 3M

C (Vol 3) - 1521, 1532, 1623, 1651-1653, Appendix 5D

C (Vol 4)- 2046

noxious (see also: Cheatgrass; Exotic plants) -

$\mathrm{H}-1,15$

I- 143,164

S- 22,85

C (Vol 2)- 433-434, 437, 459, 763, 782-785

$\mathrm{C}(\mathrm{Vol} 3)-1521,1623,1652$

$\mathrm{C}(\mathrm{Vol} 4)-2046$

Westslope cutthroat trout (see: Salmonids, key)

Wetlands-

I- 60,164

S- 74, 99

C (Vol 1) - 35, 37, 40-41, 77, 145, 147, 172, 200, 202, 231, 246, 253, 255

C (Vol 2) - 409, 479, 697, 767-769, 787-793, Appendix 3B, Appendix 3C, Appendix 3E, Appendix 3F

C (Vol 3) - 1085-1087, 1098, 1109-1110, 1128, 1161, 1314, 1318, 1320, 1365, 1431, Appendix 4A, Appendix 4B, 1525, 1540, 1554, 1565, 1570-1571, 1577,

$\mathrm{C}(\mathrm{Vol} 4)-1801,1913$ 1582-1584, 1586, 1603, 1618-1619, 1621, 1650-1651, Appendix 5F

Wildfire (see: Fire, wildfire)-

Wildland interaction/Wildland interface-

I- 15, 31-32, 130-132, 142-143, 155-157, 184

S- 57, 92 (Map 13), 135-136

C (Vol 1)- 55

C (Vol 2)- 404, 447-448, 531, 546 (Map 3.33), 560, 573, 586, 593, 603, 611, 621, 626, $636,646,656,664,855,867-869,910,916,918,961$

C (Vol 4)- 1899, 2026, 2032 
Wood products-

$\mathrm{F}$ - Appendix B

$\mathrm{H}-22$

I- 60,82

$\mathrm{S}-42-43,55$

$\mathrm{C}($ Vol 1) -84

$\mathrm{C}($ Vol 3) - 1583

C (Vol 4) — 1732, 1738-1739, 1790-1791, 1793, 1808, 1832, 1838, 1889, 1937, 1941, 1955,1986

Woody debris (see also: Coarse woody debris) -

I- $16,97,153,164,170,172,179,181,184$

$\mathrm{S}-68,74,79,89,107,116,122,140$

C (Vol 1) - 201-202, 205-206, 229

C (Vol 2)- 370, 372, 432-433, 466-467, 479-481, 487-488, 497, 500, 502, 515-516, 531, $560,646,767-768,770-773,821,823,885,888,894,896,963$, Appendix 3M

C (Vol 3) - 1074, 1084, 1086, 1099, 1101-1102, 1110, 1126, 1128, 1211, 1234, 1285, $1288,1340,1365-1367,1369,1556,1563-1565,1570,1583,1586,1601$,

Appendix 5F

$\mathbf{X}$

Xeric -

C (Vol 2)- 457, 777, 794, 802, Appendix 3A, Appendix 3F

Y

Yellowstone cutthroat trout (see: Salmonids, key)

\section{$\mathbf{Z}$}

Zonal flow (Zonal circulation) -

C (Vol 1)- 196, 312, 314

Zooplankton-

C (Vol 3) - 1107, 1278, 1432, Appendix 4A, 1510

\section{Acknowledgments}

This publication was prepared by members of the Interior Columbia Basin Ecosystem Management Project. Special thanks to Ann Marie Walker, who developed the bulk of the index; Laurieanne Riley, who developed the introduction; and Greg Bell and Rebecca Gravenmier who provided initial review. Jodi Clifford provided overall editing and compilation. 
BLM Library

Denver Federal Center

Bldg. 50, OC-521

pO. Box 25047

Denver, CO 80225

88056912

$p$

BLM LIBPARY

BLDG 50, ST-150A

DENVER FEDERAL CENTER

P.O. BOX 25047

DENVER, COLORADO 80225 
The Forest Service of the U.S. Department of Agriculture is dedicated to the principle of multiple use management of the Nation's forest resources for sustained yields of wood, water, forage, wildlife, and recreation. Through forestry research, cooperation with the States and private forest owners, and management of the National Forests and National Grasslands, it strives - as directed by Congress--to provide increasingly greater service to a growing Nation.

The U.S. Department of Agriculture (USDA) prohibits discrimination in all its programs and activities on the basis of race, color, national origin, gender, religion, age, disability, political beliefs, sexual orientation, or marital or family status. (Not all prohibited bases apply to all programs.) Persons with disabilities who require alternative means for communication of program information (Braille, large print, audiotape, etc.) should contact USDA's TARGET Center at (202) 720-2600 (voice and TDD).

To file a complaint of discrimination, write USDA, Director, Office of Civil Rights, Room 326-W, Whitten Building, 14th and Independence Avenue, SW, Washington, DC 20250-9410 or call (202) 720-5964 (voice and TDD). USDA is an equal opportunity provider and employer.

Pacific Northwest Research Station

333 S.W. First Avenue

P.O. Box 3890

Portland, Oregon 97208-3890 
